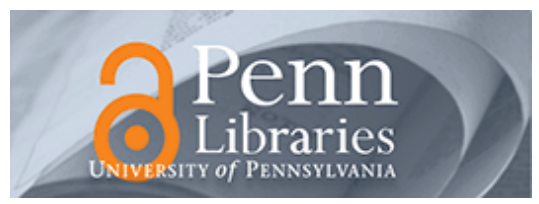

University of Pennsylvania

ScholarlyCommons

Business Economics and Public Policy Papers

Wharton Faculty Research

$12-2009$

\title{
Modeling the Birth and Death of Cartels with an Application to Evaluating Competition Policy
}

Joseph E. Harrington Jr

University of Pennsylvania

Myong-Hun Chang

Cleveland State University

Follow this and additional works at: https://repository.upenn.edu/bepp_papers

Part of the Business Commons, Economics Commons, and the Public Affairs, Public Policy and Public Administration Commons

\section{Recommended Citation}

Harrington, J. E., \& Chang, M. (2009). Modeling the Birth and Death of Cartels with an Application to Evaluating Competition Policy. Journal of the European Economic Association, 7 (6), 1400-1435.

http://dx.doi.org/10.1162/JEEA.2009.7.6.1400

At the time of publication, author Joseph E. Harrington, Jr. was affiliated with the John Hopkins University. Currently, he is a faculty member in the Business, Economy and Public Policy Department of the Wharton School at the University of Pennsylvania.

This paper is posted at ScholarlyCommons. https://repository.upenn.edu/bepp_papers/25

For more information, please contact repository@pobox.upenn.edu. 


\title{
Modeling the Birth and Death of Cartels with an Application to Evaluating Competition Policy
}

\author{
Abstract \\ One of the primary challenges to measuring the impact of antitrust or competition policy on collusion is \\ that the cartel population is unobservable; we observe only the population of discovered cartels. To \\ address this challenge, a model of cartel creation and dissolution is developed to endogenously derive \\ the populations of cartels and discovered cartels. With this theory, one can infer the impact of \\ competition policy on the population of cartels by measuring its impact on the population of discovered \\ cartels. In particular, changes in the duration of discovered cartels can be informative in assessing \\ whether a new policy is reducing the latent rate of cartels.

\section{Disciplines} \\ Business | Economics | Public Affairs, Public Policy and Public Administration

\section{Comments} \\ At the time of publication, author Joseph E. Harrington, Jr. was affiliated with the John Hopkins University. \\ Currently, he is a faculty member in the Business, Economy and Public Policy Department of the Wharton \\ School at the University of Pennsylvania.
}




\title{
MODELING THE BIRTH AND DEATH OF CARTELS WITH AN APPLICATION TO EVALUATING COMPETITION POLICY
}

Joseph E. Harrington, Jr.

Johns Hopkins University

\author{
Myong-Hun Chang \\ Cleveland State University
}

\begin{abstract}
One of the primary challenges to measuring the impact of antitrust or competition policy on collusion is that the cartel population is unobservable; we observe only the population of discovered cartels. To address this challenge, a model of cartel creation and dissolution is developed to endogenously derive the populations of cartels and discovered cartels. With this theory, one can infer the impact of competition policy on the population of cartels by measuring its impact on the population of discovered cartels. In particular, changes in the duration of discovered cartels can be informative in assessing whether a new policy is reducing the latent rate of cartels. (JEL: L13, L41)
\end{abstract}

\section{Introduction}

In recent years, numerous developments in antitrust and competition policy have occurred to fight cartels. In the European Union, many member countries have adopted more rigorous enforcement. In 1998, the European Commission published guidelines for the determination of corporate fines for price fixing which were then revised in 2006. ${ }^{1}$ In December 2005, the European Commission issued a green paper on the issue of using customer damages as a penalty, which is now a topic under intense discussion. On the other side of the Atlantic, the Antitrust Division of the U.S. Department of Justice has also adopted new policies. The revision of the Federal Sentencing Guidelines in 1991 allowed for significantly higher fines. ${ }^{2}$ With the Antitrust Criminal Penalty Enhancement and Reform Act of 2004, the statutory limit on corporate penalty was raised from $\$ 10$ million to $\$ 100$ million. And perhaps the most significant policy innovation was the 1993

The editor in charge of this paper was Xavier Vives.

Acknowledgments: We appreciate the comments of two anonymous referees, Steve Martin, Stergios Skaperdas, Rune Stenbacka, and participants at the 2006 International Industrial Organization Conference (especially Maggie Levenstein and Valerie Suslow) and the 2007 AEA meetings. The first author gratefully acknowledges the support of the National Science Foundation (SES-0516943).

E-mail addresses: Harrington: joe.harrington@jhu.edu; Chang: m.chang@csuohio.edu

1. See Wils (2007) for an analysis.

2. For a recent discussion of fines set by the U.S. Department of Justice, see Connor (2007). 
revision of the Corporate Leniency Program. ${ }^{3}$ Its apparent success led the European Commission to adopt its own program in 1996, which was then substantively revised in 2002.

With all of these policy changes-new guidelines for penalties, more aggressive enforcement, leniency programs - it is natural to ask: What effect have they had? The fundamental obstacle to addressing this question is that we do not observe the population of cartels. Due to their illegality, cartels hide themselves; we observe only the population of discovered cartels. To see the difficulties that can arise, consider a policy that impacts both the rate of cartel formation and the rate at which cartels are discovered. By both raising the discovery rate and reducing the number of cartels, the effect on the number of convictions is generally ambiguous. Hence, the number of convictions could be unchanged even though the policy is working as intended. Alternatively, the lack of change in the number of convictions could reflect the ineffectiveness of the new policy. How are we to judge the efficacy of a policy when the intended variable to be affected-the cartel rate-is not observed?

The approach of this paper is to develop a model that endogenizes the population of cartels and the population of discovered cartels and then identifies how these two populations are related. What observable change in the population of discovered cartels is informative as to what is happening with the frequency of cartels? To address this question, a population of heterogeneous industries is considered and the birth and death process for cartels is modeled. Industries are given stochastic opportunities to form a cartel and do so if it is incentive compatible. Because of random market conditions, a cartel may persist or perish because it is no longer incentive compatible to collude; they may also be discovered by the antitrust or competition authorities. Cartel formation and demise is then a stochastic process and we characterize its stationary distribution to derive a rate of cartelization for this population of industries.

Policy changes are considered that impact the probability of detection and conviction - such as the budget of the competition authority - and also those that influence the size of penalties. ${ }^{4}$ For some policy changes, we find that observing the change in the number of discovered cartels is an effective proxy for the change in the number of cartels. For other policy changes, it is not. In the latter case, a useful proxy proves to be the change in the duration of discovered cartels. In particular, if discovered cartels tend to be longer in duration after a policy change then the policy change is probably reducing cartel formation. The intuition is quite simple. If the new policy is more efficacious then its adoption will immediately

3. For a survey on leniency programs and how this work fits into the broader literature on enforcement, see Spagnolo (2008).

4. We also briefly describe some results—available on request-regarding the institution of a leniency program which impacts both the probability of paying penalties and the amount of penalties. 
cause the marginally stable cartels to collapse which, by virtue of being marginally stable, tend to be of relatively short duration. Their exit from the cartel population means they cannot be discovered (though all that is necessary for the result is that the likelihood of them being discovered is reduced by virtue of their exit). It follows that the surviving cartels are those which tend to be more stable and thus of longer duration. Because it is those cartels which make up the pool from which cartels are discovered, the average duration of discovered cartels rises in the short run in response to a more effective competition policy.

Though this project is focused on providing methods for evaluating policies designed to fight cartels, it makes a broader contribution to the general theoretical literature on collusion by modeling the birth and death process of cartels. Despite its immensity and richness, the theory of collusion focuses on a single industry in describing what conditions are conducive to collusion and what types of patterns in prices and quantities are associated with collusion. Though there is some work that endogenizes cartel formation—such as Selten (1973)—this research has not modeled the birth and death of cartels and thus cannot address the questions motivating this study. This paper shows how one can model the stochastic process determining the population of cartels and use it to generate a rate of cartelization for an economy. Independently, Miller (2009) has specified and explored an exogenous stochastic process for cartels though cartel formation and dissolution are not endogenized as in the current paper.

Finally, it is worth noting that the measurement problem motivating this paper is not unique to price-fixing; it arises as well for other forms of criminal activity including tax evasion, extortion, blackmail, and kidnapping. These types of crimes are common in that they are often not reported; either because the victim does not know they are being victimized (such as with price-fixing and tax evasion) or the victim does not have an incentive to report (due to the threat of some punishment). This creates the challenge of measuring the rate of criminal activity and assessing the impact of a policy on the crime rate. One approach to measuring the crime rate is to engage in random sampling. Although that may work with tax evasion, it would be difficult to randomly sample industries for collusion or small businesses for extortion. There is then a fundamental challenge in measuring the latent rate of criminal activity with data based on non-random sampling.

After reviewing the model in Section 2, the cartel formation decision is characterized in Section 3. Aggregating cartel creation and dissolution across a heterogeneous pool of industries, the stationary distribution on cartels is constructed in Section 4. The main results are in Section 5.

\section{Model}

The objective is to construct a model in which some industries collude and some do not, some cartels collapse and some do not, some cartels are caught and some 
are not. As we are going to consider a population of industries, the model of each industry is kept simple by using a Prisoners' Dilemma formulation. For each industry, there is a stochastic realization of a market's profitability that is summarized by the variable $\pi \geq 0$. If firms are colluding then each firm earns $\pi$; if they are not colluding then each earns $\alpha \pi$, where $\alpha \in[0,1) . \pi$ has a continuously differentiable cumulative distribution function (c.d.f.) $H:[\underline{\pi}, \bar{\pi}] \rightarrow[0,1]$ where $0<\underline{\pi}<\bar{\pi}$ and $\bar{\pi}$ may be finite or infinite. $h(\cdot)$ denotes the associated density function. Let $\mu \equiv \int \pi h(\pi) d \pi$ denote its finite mean. As in Rotemberg and Saloner (1986), $\pi$ is observed prior to firms deciding how to behave. If all other firms are colluding, the profit a firm earns by deviating is $\eta \pi$ where $\eta>1$. Let $\delta \in(0,1)$ denote the common discount factor. Note that the Bertrand price game is represented by $(\alpha, \eta)=(0, n)$ where $n$ is the number of firms. The Cournot quantity game with linear demand and cost functions in which firms collude at the joint profit maximum can be represented as $(\alpha, \eta)=\left(4 n /(n+1)^{2},(n+1)^{2} / 4 n\right)$.

At the start of each period, an industry is either cartelized or not. If it was cartelized at the end of the previous period then it is currently cartelized. If it was not cartelized at the end of the previous period then with probability $\kappa \in(0,1)$ it has an opportunity to do so. ${ }^{5}$ Given the realization of $\pi$, if firms either are not cartelized or if collusion is not incentive compatible then each firm earns $\alpha \pi$. If there is a cartel and collusion is incentive compatible then each earns $\pi$.

At the end of the period, there is the random event whereby the competition authority may pursue an investigation; this can only occur if firms colluded in the current or previous period. ${ }^{6}$ Let $\sigma \in[0,1)$ denote the probability that firms are discovered and convicted. In that case, each firm incurs a penalty of $F /(1-\delta)$ (so that $F$ is the per-period penalty). For reasons of tractability, the standard assumption in repeated game models with a competition authority is that the penalty is fixed and thus independent of the severity of the offense. ${ }^{7}$ Given that there is only one collusive price in our model, severity necessarily refers to the number of periods that firms colluded. Allowing the penalty to depend on severity would then mean having, for each cartel, a state variable which is the length of time of collusion. The introduction of that state variable would be a serious complication of the model and suggests why, in previous models, the penalty is instead assumed fixed. We have, however, found a tractable specification that

5. That $\kappa<1$ may be because cartelization requires having a set of managers willing to break the law or that feel they can communicate and trust each other or an opportunity arises to communicate without much risk of being caught.

6. It is natural to suppose that the likelihood of being caught declines with the length of time since firms colluded. For example, discovery may be due to suspicions of uninvolved employees but they are less likely to become suspicious as the flow of fresh evidence is cut off due to the cartel no longer operating. Having the decay occur immediately after one period is a simplification that improves tractability since otherwise it would require introducing a state variable which is the time at which firms last colluded.

7. For a review of some of this work, see Spagnolo (2008). 
allows the penalty to depend on the average severity: The penalty is assumed to be proportional to the average increase in profit from being cartelized (rather than the realized increase in profit). If $Y$ denotes the average per period profit from being in the "cartel state" then $F=\gamma(Y-\alpha \mu)$ where $\gamma>0$. This specification avoids the introduction of a state variable but still allows the penalty to be sensitive to the (average) extent of collusion. In sum, the competition policy parameters are $(\sigma, \gamma)$ which are, respectively, the probability of paying penalties and the penalty multiple.

Whenever a cartel is shut down-whether due to internal collapse or having been successfully prosecuted - the industry may re-cartelize in the future. Specifically, it has an opportunity to do so with probability $\kappa$ in each period that it is not currently colluding. Alternatively, one could imagine having two distinct probabilities - one to reconstitute collusion after a firm cheated (the probability of moving from the punishment to the cooperative phase) and another to reform the cartel after having been convicted. For purposes of parsimony and tractability, those two probabilities are assumed to be the same; we have no reason to think that results are sensitive to this assumption.

The modeling of collusion here differs from how it is typically done. The standard approach presumes that firms are always coordinating; even when they are competing, it is a coordinated punishment in response to suspected cheating. In a perfect monitoring setting such as Rotemberg and Saloner (1986), it may require adjusting the collusive price so as to maintain cartel stability. Or, in an imperfect monitoring setting, it may require periodic shifts to distinctly lower prices. But, at all times, firms are coordinating their behavior. We do not believe that is always a reasonable representation of reality. There are many well-documented episodes in which a cartel truly collapses in the sense that coordination stops and what emerges from the ashes is competition. Of course, coordination may start up again but it need not be immediate and the prospect of it re-starting may be reasonably viewed as uncertain from the perspective of firms.

In modeling a population of industries, it is compelling to allow industries to vary in terms of cartel stability. For this purpose, industries are assumed to differ in terms of the parameter $\eta$. If one takes this assumption literally, it can be motivated by heterogeneity in the elasticity of firm demand or the number of firms (as with the Bertrand price game). Our intent is not to be literal but rather to think of this as a parsimonious way in which to encompass industry heterogeneity. Let the c.d.f. on industry types be represented by the continuously differentiable function $G:[\eta, \bar{\eta}] \rightarrow[0,1]$ where $1<\eta<\bar{\eta} \cdot g(\cdot)$ denotes the associated density function. The appeal of having heterogeneity with respect to $\eta$ is that it is a parameter which influences the frequency of collusion but does not directly affect the value of the firm's profit stream since, in equilibrium, firms do not cheat; this makes for an easier analysis. In Section 5.3, we discuss the robustness of our results with respect to other forms of industry heterogeneity. 


\section{Equilibrium Cartel Formation}

Characterization of the stationary distribution on cartels is a three-step procedure. First, the equilibrium conditions for a cartel to be stable in a type- $\eta$ industry are characterized in this section. This result is then used in the ensuing section where the stationary distribution on the sub-population of type- $\eta$ industries is first characterized and then that result is integrated over all values of $\eta$ to derive the aggregate stationary distribution.

\subsection{Existence of an Equilibrium}

A collusive strategy for a type- $\eta$ industry entails colluding when $\pi$ is sufficiently low and not colluding otherwise. The logic is as in Rotemberg and Saloner (1986). When $\pi$ is high, the incentive to deviate is strong since a firm increases current profit by $(\eta-1) \pi$. Given that $\pi$ is i.i.d., the future payoff is independent of the current realization of $\pi$. Because the payoff to cheating is increasing in $\pi$ while the future payoff is independent of $\pi$, the incentive compatibility of collusion is more problematic when $\pi$ is higher. Depending on the parameter values, it is possible that collusion is not incentive compatible for any $\pi \in[\underline{\pi}, \bar{\pi}]$, in which case those industries never cartelize. Similarly, it may be the case that collusion is incentive compatible for all $\pi \in[\underline{\pi}, \bar{\pi}]$, in which case such cartels are never subject to internal collapse and are only shut down by the authorities.

Suppose firms are able to collude for at least some realizations of $\pi$. Let $W^{o}$ and $Y^{o}$ denote the payoff when the industry is not cartelized and is cartelized, respectively. If not cartelized then, with probability $\kappa$, firms have an opportunity to cartelize with resulting payoff $Y^{o}$. With probability $1-\kappa$, firms do not have such an opportunity and continue to compete. In that case, each firm earns current expected profit of $\alpha \mu$ and a future value of $W^{o}$. Thus, the payoff when not colluding is defined recursively by

$$
W^{o}=(1-\kappa)\left(\alpha \mu+\delta W^{o}\right)+\kappa Y^{o} .
$$

It will be easier to work with re-scaled payoffs, so define

$$
W \equiv(1-\delta) W^{o}, \quad Y \equiv(1-\delta) Y^{o} .
$$

Multiplying both sides of equation (1) by $1-\delta$ and re-arranging yields

$$
W=(1-\kappa)[(1-\delta) \alpha \mu+\delta W]+\kappa Y,
$$

and then solving for $W$ yields

$$
W=\frac{(1-\kappa)(1-\delta) \alpha \mu+\kappa Y}{1-\delta(1-\kappa)} .
$$


Note also that the incremental value to being in the cartelized state is

$$
Y-W=Y-\left(\frac{(1-\kappa)(1-\delta) \alpha \mu-\kappa Y}{1-\delta(1-\kappa)}\right)=\frac{(1-\kappa)(1-\delta)(Y-\alpha \mu)}{1-\delta(1-\kappa)}
$$

Suppose firms are cartelized and $\pi$ is realized. The incentive compatibility constraint (ICC) is

$$
(1-\delta) \pi+\delta[(1-\sigma) Y+\sigma(W-F)] \geq(1-\delta) \eta \pi+\delta(W-\sigma F),
$$

which can be solved for $\pi$ to obtain

$$
\pi \leq \frac{\delta(1-\sigma)(Y-W)}{(1-\delta)(\eta-1)} \equiv \varphi(Y, \eta) .
$$

Collusion is stable when the profit realization is sufficiently low. Given that all other firms collude, a firm earns $\pi$ by also colluding and it has an expected continuation payoff of $(1-\sigma) Y+\sigma(W-F)$, since with probability $\sigma$ it is caught and convicted, in which case the industry shifts to the non-cartel state and each firm pays a penalty of $F{ }^{8}$ Note that the expected penalty does not impact the ICC because it is unaffected by whether a firm cheats or colludes. The presumption is that discovery depends only on whether firms attempted to coordinate this period and not on whether such coordination was successful (that is, a firm does not believe its act of cheating alters the likelihood of paying penalties in the current period). This is clearly a simplification and indeed there have been analyses modeling how the realized price path influences the likelihood of detection and feeds back to impact the optimal cartel price path (Harrington 2004, 2005; Harrington and Chen 2006).

The expected (rescaled) payoff to being cartelized can now be recursively defined. Given an industry is cartelized and given a profit realization $\pi \leq \varphi(Y, \eta)$, each firm earns profit of $\pi$ and the cartel is caught with probability $\sigma$ in which case each receives the future non-collusive payoff $W$ less the penalty $F$; and if not caught each earns the future collusive value $Y$. If instead $\varphi(Y, \eta)<\pi$ then the cartel collapses so each firm earns $\alpha \pi$ and the future value is $W$ less expected penalties. ${ }^{9}$ The following equation then defines the implied collusive value, $\psi(Y)$, when firms perceive it to be $Y$.

\footnotetext{
8. We will later substitute $\gamma(Y-\alpha \mu)$ for $F$.

9. One might expect the probability of being caught to be lower if firms are not currently colluding (though did collude in the previous period). This we consider a reasonable approximation in that the difference in probability is likely to be small and making this assumption simplifies the analysis.
} 


$$
\begin{aligned}
\psi(Y)= & \int_{\underline{\pi}}^{\frac{\delta(1-\sigma)(Y-W)}{(1-\delta)(\eta-1)}}[(1-\delta) \pi+\delta Y-\delta \sigma(Y-W)] h(\pi) d \pi \\
& +\int_{\frac{\delta(1-\sigma)(Y-W)}{(1-\delta)(\eta-1)}}^{\bar{\pi}}[(1-\delta) \alpha \pi+\delta W] h(\pi) d \pi-\delta \sigma F .
\end{aligned}
$$

To derive an expression in only one unknown, $Y$, substitute for $(Y-W)$ using equation (3), substitute for $W$ using equation (2), and replace $F$ with $\gamma(Y-\alpha \mu)$ :

$$
\begin{aligned}
& \psi(Y)= \\
& \quad \int_{\underline{\pi}}^{\frac{\delta(1-\sigma)(1-\kappa)(Y-\alpha \mu)}{[1-\delta(1-\kappa)](\eta-1)}}\left[(1-\delta) \pi+\delta Y-\left(\frac{\delta \sigma(1-\kappa)(1-\delta)(Y-\alpha \mu)}{1-\delta(1-\kappa)}\right)\right] h(\pi) d \pi \\
& +\int_{\frac{\delta(1-\sigma)(1-\kappa)(Y-\alpha \mu)}{[1-\delta(1-\kappa)](\eta-1)}}^{\bar{\pi}}\left[(1-\delta) \alpha \pi+\delta\left(\frac{(1-\kappa)(1-\delta) \alpha \mu+\kappa Y}{1-\delta(1-\kappa)}\right)\right] h(\pi) d \pi \\
& -\delta \sigma \gamma(Y-\alpha \mu) .
\end{aligned}
$$

A fixed point of $\psi$ is an equilibrium value of $Y$. That is, given an anticipated collusive value $Y$, the resulting equilibrium behavior results in that same value when firms are cartelized.

As an initial step to exploring the set of fixed points, first note that $\psi(\alpha \mu)=$ $\alpha \mu$. Hence, one fixed point to $\psi$ is the degenerate solution without collusion. It is straightforward to show $\psi(\mu) \leq \mu$ and, if $\sigma>0$ and $\gamma>0$, then $\psi(\mu)<\mu$. The issue is whether there is another fixed point, in which case $Y>\alpha \mu$, and thus firms cartelize with positive probability. Theorem 1 will help us establish when that is the case.

THEOREM 1. Assume $\kappa /(1-\delta(1-\kappa))>\sigma \gamma$. Then:

(i) $\psi:[\alpha \mu, \mu] \rightarrow[\alpha \mu, \mu]$;

(ii) $\psi^{\prime}(Y) \geq 0$ for all $Y \in[\alpha \mu, \mu)$;

(iii) if $\varphi(Y, \eta) \in(\underline{\pi}, \bar{\pi})$ then $\psi^{\prime}(Y)>0$.

All proofs are in the Appendix.

Thus, if the probability of discovery and conviction, $\sigma$, and/or the penalty multiple, $\gamma$, are sufficiently low then $\psi$ continuously maps $[\alpha \mu, \mu]$ into itself and is an increasing function of $Y$. From here on, assume

$$
\frac{\kappa}{1-\delta(1-\kappa)}>\sigma \gamma .
$$

Define $Y^{*}$ to be the maximal fixed point of $\psi$,

$$
Y^{*}(\eta) \equiv \max \{Y \in[\alpha \mu, \mu]: \psi(Y ; \eta)=Y\} .
$$


A collusive solution is said to exist when $Y^{*}>\alpha \mu$. The next result shows that, when the probability of having to pay penalties is sufficiently low, a cartel forms with positive probability when $\eta$ is sufficiently low. Otherwise, a cartel never forms.

THEOREM 2. If $\sigma$ is sufficiently close to zero then there exists $\hat{\eta}>1$ such that (a) $Y^{*}(\eta) \in(\alpha \mu, \mu]$ if $\eta \in(1, \hat{\eta}]$ and (b) $Y^{*}(\eta)=\alpha \mu$ if $\eta>\hat{\eta}$.

From here on, assume $\sigma$ is sufficiently low and $\eta$ is sufficiently close to one so that a cartel forms for sufficiently low values of $\eta$ (that is, $\hat{\eta}>\eta$ ) and, in addition, does not form for sufficiently high values of $\eta$ (that is, $\bar{\eta}$ is sufficiently high so that $\hat{\eta}<\bar{\eta}$ ).

If there are multiple interior solutions to equation (6), we make the usual selection that firms achieve the equilibrium with the highest value, which is $Y^{*}(\eta)$. Given $Y^{*}(\eta)$, define $\varphi^{*}(\eta)$ as the maximum profit realization such that the cartel is stable,

$$
\varphi^{*}(\eta) \equiv \frac{\delta(1-\sigma)(1-\kappa)\left[Y^{*}(\eta)-\alpha \mu\right]}{[1-\delta(1-\kappa)](\eta-1)} .
$$

$\varphi^{*}(\eta)$ is a measure of cartel stability because the cartel is stable if and only if $\pi \leq \varphi^{*}(\eta)$. This can be seen more clearly by noting that the probability a cartel survives in any period is $(1-\sigma) H\left(\varphi^{*}(\eta)\right)$ which is non-decreasing in $\varphi^{*}(\eta)$.

\subsection{Comparative Statics}

Theorem 3 provides some intuitive comparative statics regarding the value to cartelizing, $Y^{*}(\eta)$, and the measure of cartel stability, $\varphi^{*}(\eta)$.

THEOREM 3. $Y^{*}(\eta)$ is non-increasing in $\sigma, \gamma$, and $\eta$. If $Y^{*}(\eta) \in(\alpha \mu, \mu)$ then $Y^{*}(\eta)$ is decreasing in $\sigma$ and $\gamma$. If $\varphi^{*}(\eta) \in(\underline{\pi}, \bar{\pi})$ then $Y^{*}(\eta)$ is decreasing in $\eta$ and $\varphi^{*}(\eta)$ is decreasing in $\sigma, \gamma, \eta$, and $\alpha$.

When competition policy is made tougher-as reflected in a higher value for $\sigma$ or $\gamma$-the value to forming a cartel, $Y^{*}(\eta)$, is reduced. There is both a direct effect and an indirect effect underlying this result. The direct effect is that the expected penalty is higher-by making conviction more likely or increasing the penalty multiple - and this lowers the collusive value, holding firm behavior fixed. This higher expected penalty induces an indirect (behavioral) effect as it reduces the range of profit realizations for which the cartel is stable (that is, $\varphi^{*}(\eta)$ is decreasing in $\sigma$ and $\gamma$ ). Therefore, firms expect a shorter duration from forming a cartel and this also serves to reduce the collusive value. Hence, both the direct 
and indirect effects work to reduce the value to being cartelized and the stability of the cartel. With regards to the effect of the industry type $\eta$, only the indirect effect is operative. There is a greater payoff to cheating for an industry with a higher value for $\eta$ and this translates into a less stable cartel and a lower value to being cartelized. A final parameter of interest is the non-collusive profit rate which is controlled by $\alpha$. As $\alpha$ rises, the expected incremental profit from colluding, $(1-\alpha) \mu$, is reduced, and the cartel is made less stable because the punishment from cheating declines. A higher value for $\alpha$ then weakens the incentive to form a cartel and reduces cartel stability. ${ }^{10}$

Recall from Theorem 2 that if $\eta$ is sufficiently high then an industry will not cartelize for any profit state; only industries for which $\eta \leq \hat{\eta}$ can potentially cartelize. The next result shows that when competition policy is tougher or the non-collusive profit is higher, there is a smaller set of industries that are able to cartelize. The intuition is the same as with Theorem 3.

TheOREM 4. $\hat{\eta}$ is decreasing in $\sigma, \gamma$, and $\alpha$.

\section{Stationary Distribution on Cartels}

Using the preceding analysis, the stochastic process by which cartels are born and die (either through internal collapse or being caught) is characterized in this section. The random events driving this process are: (i) the opportunity to cartelize; (ii) the profit conditions; and (iii) detection by the competition authority. We initially characterize the stationary distribution for the subset of type- $\eta$ industries. The stationary distribution for the entire population of industries is then characterized by integrating the distributions for the type- $\eta$ industries.

Section 3 characterized behavior for a specific industry. There it was shown that if a type- $\eta$ industry was not cartelized at the end of the previous period then it will cartelize and collude in the current period with probability $\kappa H\left(\varphi^{*}(\eta)\right)$. If a type- $\eta$ industry was cartelized at the end of the previous period then it will still be cartelized at the end of this period with probability $(1-\sigma) H\left(\varphi^{*}(\eta)\right)$. Suppose there is a continuum of type- $\eta$ industries with independent realizations of the stochastic events each period. The task is to characterize the stationary distribution with regards to the frequency and duration of cartels.

Let $\beta(l ; \eta)$ denote the proportion of type- $\eta$ industries with cartels of length $l \in\{0,1,2, \ldots\} . l=0$ means firms are not cartelized so that $1-\beta(0 ; \eta)$ is the fraction of cartels among type- $\eta$ industries. To reduce the notational burden,

10. The collusive value can be shown to be increasing in the non-collusive profit rate (which is controlled by $\alpha$ ) but that is not very informative because the collusive value is also based on periods during which firms earn the non-collusive profit. More informative is that the incremental expected gain from colluding, $Y^{*}(\eta ; \alpha)-\alpha \mu$, can be shown to be decreasing in $\alpha$. It is the latter property that results in $\varphi^{*}(\eta ; \alpha)$ being decreasing in $\alpha$. 
$\eta$ will often be suppressed. The stationary distribution is defined by the following set of equations:

$$
\begin{aligned}
\beta(0 ; \eta)= & \beta(0 ; \eta)\left[(1-\kappa)+\kappa\left(1-H\left(\varphi^{*}\right)\right)+\kappa \sigma H\left(\varphi^{*}\right)\right] \\
& +[1-\beta(0 ; \eta)]\left[\left(1-H\left(\varphi^{*}\right)\right)+\sigma H\left(\varphi^{*}\right)\right], \\
\beta(1 ; \eta)= & \beta(0 ; \eta) \kappa(1-\sigma) H\left(\varphi^{*}\right), \\
\beta(l ; \eta)= & \beta(l-1 ; \eta)(1-\sigma) H\left(\varphi^{*}\right), \quad l \in\{2,3, \ldots\} .
\end{aligned}
$$

Note that $\eta$ enters through $\varphi^{*}$. Considering the right-hand side of equation (9), fraction $\beta(0 ; \eta)$ of type- $\eta$ industries were not cartelized in the previous period. Out of those industries, a fraction $1-\kappa$ will not have the opportunity to cartelize and thus will not collude in the current period. A fraction $\kappa\left(1-H\left(\varphi^{*}\right)\right)$ will have the opportunity but, due to a high profit realization, find it is not incentive compatible to collude, while a fraction $\kappa \sigma H\left(\varphi^{*}\right)$ will cartelize but then are discovered by the authorities. Of the industries that were colluding in the previous period, which have mass $1-\beta(0 ; \eta)$, fraction $1-H\left(\varphi^{*}\right)$ will collapse for internal reasons and a fraction $\sigma H\left(\varphi^{*}\right)$ will instead be caught by the authorities and thus shut down. Turning to equation (10), an industry can go from competing to colluding by being presented with the opportunity to cartelize, which occurs with probability $\kappa$, and having a sufficiently low profit realization, which occurs with probability $H\left(\varphi^{*}\right)$. Finally, an existing cartel continues to collude-which means if it is of length $l-1$ then its length grows to $l$-if the profit realization is sufficiently low and it is not discovered and convicted; the joint probability of that event is $(1-\sigma) H\left(\varphi^{*}\right)$ which gives us equation (11).

Solving equation (9) for $\beta(0 ; \eta)$, we get

$$
\beta(0 ; \eta)=\frac{1-(1-\sigma) H\left(\varphi^{*}\right)}{1-(1-\kappa)(1-\sigma) H\left(\varphi^{*}\right)} .
$$

In the steady-state, the fraction of cartels among type- $\eta$ industries is then

$$
1-\beta(0 ; \eta)=\frac{\kappa(1-\sigma) H\left(\varphi^{*}\right)}{1-(1-\kappa)(1-\sigma) H\left(\varphi^{*}\right)} .
$$

Next note that

$$
\beta(l ; \eta)=\beta(0 ; \eta) \kappa[H(\varphi)(1-\sigma)]^{l}, \quad l \geq 1 .
$$

A mass $\beta(0 ; \eta) \kappa$ of industries get the chance to form a cartel and a fraction of $[H(\varphi)(1-\sigma)]^{l}$ will still be cartelized $l$ periods later. Using equation (12), we can substitute for $\beta(0 ; \eta)$ in equation (14) and obtain

$$
\beta(l ; \eta)=\frac{\left[1-(1-\sigma) H\left(\varphi^{*}\right)\right] \kappa\left[H\left(\varphi^{*}\right)(1-\sigma)\right]^{l}}{1-(1-\kappa)(1-\sigma) H\left(\varphi^{*}\right)}, \quad l \geq 1 .
$$


Next define the probability distribution over cartel length among cartels, $\{f(l ; \eta)\}_{l=1}^{\infty}$. For $l \geq 1$,

$$
\begin{aligned}
f(l ; \eta) \equiv & \frac{\beta(l ; \eta)}{1-\beta(0 ; \eta)} \\
= & \left(\frac{\left[1-(1-\sigma) H\left(\varphi^{*}\right)\right] \kappa\left[H\left(\varphi^{*}\right)(1-\sigma)\right]^{l}}{1-(1-\kappa)(1-\sigma) H\left(\varphi^{*}\right)}\right) \\
& \times\left(\frac{\kappa(1-\sigma) H\left(\varphi^{*}\right)}{1-(1-\kappa)(1-\sigma) H\left(\varphi^{*}\right)}\right)^{-1} \\
= & {\left[1-(1-\sigma) H\left(\varphi^{*}\right)\right]\left[(1-\sigma) H\left(\varphi^{*}\right)\right]^{l-1} } \\
= & {\left[(1-\sigma) H\left(\varphi^{*}\right)\right]^{l-1}-\left[(1-\sigma) H\left(\varphi^{*}\right)\right]^{l} . }
\end{aligned}
$$

The average cartel length is then

$$
\sum_{l=1}^{\infty} l f(l ; \eta)=\frac{1}{1-(1-\sigma) H\left(\varphi^{*}\right)} .
$$

Finally, the derivation of the entire population of industries is performed by integrating the type- $\eta$ distribution over types, $\eta \in[\underline{\eta}, \bar{\eta}] .\{\tilde{\beta}(l)\}_{l=0}^{\infty}$ denotes the stationary distribution on cartel length, where

$$
\tilde{\beta}(l)=\int_{\underline{\eta}}^{\bar{\eta}} \beta(l ; \eta) g(\eta) d \eta, \quad l=0,1,2, \ldots
$$

Of particular relevance is the frequency with which industries are cartelized,

$$
\begin{aligned}
1-\tilde{\beta}(0) & =\int_{\underline{\eta}}^{\hat{\eta}}[1-\beta(0 ; \eta)] g(\eta) d \eta \\
& =\int_{\underline{\eta}}^{\hat{\eta}}\left[\frac{\kappa(1-\sigma) H\left(\varphi^{*}(\eta)\right)}{1-(1-\kappa)(1-\sigma) H\left(\varphi^{*}(\eta)\right)}\right] g(\eta) d \eta,
\end{aligned}
$$

and the frequency of discovered cartels is

$$
\sigma[1-\tilde{\beta}(0)]=\sigma \int_{\underline{\eta}}^{\hat{\eta}}\left[\frac{\kappa(1-\sigma) H\left(\varphi^{*}(\eta)\right)}{1-(1-\kappa)(1-\sigma) H\left(\varphi^{*}(\eta)\right)}\right] g(\eta) d \eta .
$$

\section{Evaluating the Impact of Competition Policy}

\subsection{Introduction}

In the firms' problem, government policy enters through the penalty multiple, $\gamma$, and the probability of detection and conviction, $\sigma$. Of relevance is that $\gamma$ 
and $\sigma$ represent firms' perception of the determination of penalties (as modeled by $\gamma$ ) and the probability of paying penalties (as modeled by $\sigma$ ). Thus, from the perspective of the competition authority, $\gamma$ and $\sigma$ are latent parameters influenced by policy. For example, the Antitrust Division of the U.S. Department of Justice may increase the amount of resources used to prosecute cartels but exactly how this will impact the probability that firms assign to being caught and convicted is unknown. Or the European Commission may alter the formula for calculating penalties, as it did with the 2006 Guidelines, but how this change translates into the perception of firms regarding the determination of penalties is unclear. In fact, the "formula" for calculating penalties involves subjective factors-such as the "seriousness of the offense"-and this leaves room for subjective beliefs.

The starting point to the exercises conducted in this section is that the competition authority makes a policy change that has an unobserved and uncertain impact on $\gamma$ or $\sigma$. Our first task is to characterize how a change in $\gamma$ or $\sigma$ affects the rate of cartel formation. With those results, a competition authority could deduce the impact of a new policy if it knew what the new values were for the latent parameters $\gamma$ and $\sigma$. However, it is presumed not to have that information. We then investigate whether the change in the cartel rate (caused by the unobserved change in $\gamma$ or $\sigma$ ) can be inferred by examining changes in the observable population of discovered cartels. The questions are then as follows: (1) What is the effect of a change in a latent policy parameter on the number of cartels? (2) Can the effect on the number of cartels be inferred from the change in the population of discovered cartels?

\subsection{Main Results}

When penalties for collusion are increased, Theorem 5 reports that there are both fewer cartels and fewer discovered cartels, as reflected in the stationary distribution. This is not surprising because more severe penalties result in less stable cartels-as $\varphi^{*}(\eta)$ is decreasing in $\gamma$ by Theorem 3-which means that a given industry is in the cartelized state a smaller fraction of the time. Furthermore, by Theorem $4, \hat{\eta}$ is decreasing in $\gamma$ which means that a smaller fraction of industries are ever able to cartelize.

THEOREM 5. The rate of cartel formation, $1-\tilde{\beta}(0)$, and the rate of discovered cartels, $\sigma[1-\tilde{\beta}(0)]$, are decreasing in the severity of penalties, $\gamma$.

Let us now use these results to infer the impact of a new policy on the unobserved rate of cartels by observing the rate of discovered cartels. (This exercise is straightforward but we go through it in preparation for a later discussion.) Suppose there is a change in the penalty policy which, unbeknownst to the competition authority, results in a change in $\gamma$ from $\gamma^{\prime}$ to $\gamma^{\prime \prime}$. If $\gamma^{\prime \prime}>\gamma^{\prime}$ then the policy has 
intensified penalties (as perceived by firms) in which case the cartel rate falls. Or the policy might have had some perverse consequences so that $\gamma^{\prime \prime}<\gamma^{\prime}$ and the cartel rate rises. ${ }^{11}$ Or perhaps the policy change is totally ineffective so that $\gamma^{\prime \prime}=\gamma^{\prime}$. This may arise if the maximum penalty is increased but proves not to affect expected penalties.

To infer the policy impact on the cartel rate from the discovered cartel rate, consider the ratio of the new to the old steady-state discovered cartel rate,

$$
\frac{\sigma\left[1-\tilde{\beta}\left(0 ; \gamma^{\prime \prime}\right)\right]}{\sigma\left[1-\tilde{\beta}\left(0 ; \gamma^{\prime}\right)\right]}=\frac{1-\tilde{\beta}\left(0 ; \gamma^{\prime \prime}\right)}{1-\tilde{\beta}\left(0 ; \gamma^{\prime}\right)} .
$$

In response to a change in the penalty parameter, the percentage change in the cartel rate equals the percentage change in the discovered cartel rate so the latter can be used to infer the former. Thus, if there are fewer discovered cartels after the policy change, one can infer that there are fewer cartels.

Next consider the effect of a policy change that is designed to affect the probability of discovery and conviction. Although a tougher enforcement policy that is efficacious-as reflected in a higher value for $\sigma$-reduces the cartel rate, its impact on the rate of discovered cartels is ambiguous.

THEOREM 6. The rate of cartel formation, $1-\tilde{\beta}(0)$, is decreasing in $\sigma$ but the effect of $\sigma$ on the rate of discovered cartels, $\sigma[1-\tilde{\beta}(0)]$, is ambiguous (that is, its sign depends on parameter values).

To understand why $\sigma$ has an ambiguous effect on the rate of discovered cartels, consider raising $\sigma$ from $\sigma^{\prime}$ to $\sigma^{\prime \prime}$. The change in the rate of discovered cartels equals

$$
\sigma^{\prime \prime} \int_{\underline{\eta}}^{\hat{\eta}\left(\sigma^{\prime \prime}\right)}\left[1-\beta\left(0 ; \eta, \sigma^{\prime \prime}\right)\right] g(\eta) d \eta-\sigma^{\prime} \int_{\underline{\eta}}^{\hat{\eta}\left(\sigma^{\prime}\right)}\left[1-\beta\left(0 ; \eta, \sigma^{\prime}\right)\right] g(\eta) d \eta .
$$

This can be re-arranged so that the frequency of discovered cartels goes up if and only if

$$
\begin{aligned}
\int_{\underline{\eta}}^{\hat{\eta}\left(\sigma^{\prime \prime}\right)}\left[\sigma^{\prime \prime}\left(1-\beta\left(0 ; \eta, \sigma^{\prime \prime}\right)\right)\right. & \left.-\sigma^{\prime}\left(1-\beta\left(0 ; \eta, \sigma^{\prime}\right)\right)\right] g(\eta) d \eta \\
& >\sigma^{\prime} \int_{\hat{\eta}\left(\sigma^{\prime \prime}\right)}^{\hat{\eta}\left(\sigma^{\prime}\right)}\left[1-\beta\left(0 ; \eta, \sigma^{\prime}\right)\right] g(\eta) d \eta .
\end{aligned}
$$

11. Cyrenne (1999), Spagnolo (2000), Harrington (2004), and Chen and Harrington (2007) show how antitrust policy can have the perverse effect of making collusion more stable. 
The right-hand side term is the reduction in discovered cartels because the marginally stable cartels no longer form (or, if they did form, they now collapse) and thus are not there to be caught. The left-hand side is the change in the rate of discovered cartels among those cartels that continue to form in spite of the higher chance of paying penalties.

Depending on $\sigma^{\prime}$ and $\sigma^{\prime \prime}$, equation (15) could either hold or not hold so that a rise in the probability of detection could either raise or lower the number of discovered cartels. If the initial probability of detection, $\sigma^{\prime}$, is sufficiently low then few cartels are being discovered and thus an increase to $\sigma^{\prime \prime}$ means more discovered cartels. In contrast, if $\sigma^{\prime}$ is close to one then the reduced cartel rate from raising $\sigma$ dominates the higher discovery rate so that there are fewer discovered cartels (details are in the proof of Theorem 6).

It is then problematic inferring the effect of a policy change on the latent cartel rate by measuring what has happened to the observed rate of discovered cartels. For example, a rise in the rate of discovered cartels is consistent with both the probability of detection having increased (and thus there are fewer cartels) and having decreased (and thus there are more cartels). However, as shown subsequently, one can derive an observable and unambiguous implication of a more effective detection policy by examining changes in the duration of discovered cartels.

The stationary distribution on cartel length, conditional on being cartelized $(l \geq 1)$, is

$$
\tilde{f}(l) \equiv \frac{\int_{\underline{\eta}}^{\hat{\eta}} \beta(l ; \eta) g(\eta) d \eta}{\int_{\underline{\eta}}^{\hat{\eta}}[1-\beta(0 ; \eta)] g(\eta) d \eta} .
$$

Perform the following steps:

$$
\begin{aligned}
\tilde{f}(l) & =\int_{\underline{\eta}}^{\hat{\eta}(\sigma)}\left[\frac{\beta(l ; \eta) g(\eta) d \eta}{\int_{\underline{\eta}}^{\hat{\eta}(\sigma)}\left[1-\beta\left(0 ; \eta^{\prime}\right)\right] g\left(\eta^{\prime}\right) d \eta^{\prime}}\right] \\
& =\int_{\underline{\eta}}^{\hat{\eta}(\sigma)}\left(\frac{\beta(l ; \eta, \sigma)}{1-\beta(0 ; \eta, \sigma)}\right)\left[\frac{(1-\beta(0 ; \eta, \sigma)) g(\eta)}{\int_{\underline{\eta}}^{\hat{\eta}(\sigma)}\left[1-\beta\left(0 ; \eta^{\prime}, \sigma\right)\right] g\left(\eta^{\prime}\right) d \eta^{\prime}}\right] d \eta \\
& =\int_{\underline{\eta}}^{\hat{\eta}(\sigma)} f(l ; \eta)\left[\frac{(1-\beta(0 ; \eta, \sigma)) g(\eta)}{\int_{\underline{\eta}}^{\hat{\eta}(\sigma)}\left[1-\beta\left(0 ; \eta^{\prime}, \sigma\right)\right] g\left(\eta^{\prime}\right) d \eta^{\prime}}\right] d \eta .
\end{aligned}
$$

$\tilde{f}(l)$ is then a weighted average of $f(l ; \eta)$, where the weight assigned to $f(l ; \eta)$ is the fraction of all cartels that are of type $\eta$. 
In considering the impact of raising $\sigma$ from $\sigma^{\prime}$ to $\sigma^{\prime \prime}$ on $\{\tilde{f}(l)\}_{l=1}^{\infty}$, let us break it apart into short-run and long-run effects. The short-run effect on the distribution over the duration of discovered cartels is from the immediate collapse of some cartels upon the institution of a more aggressive detection and conviction policy. The long-run effect is the change in the distribution on duration as it converges to the new stationary distribution. This can be made more concrete. Because $\hat{\eta}$ is decreasing in $\sigma$, cartels for which $\eta \in\left(\hat{\eta}\left(\sigma^{\prime \prime}\right), \hat{\eta}\left(\sigma^{\prime}\right)\right]$ are no longer stable (for any profit realizations) after $\sigma$ is raised and thereby immediately collapse. Thus, the policy change induces an immediate shift in the mass of cartels from

$$
\int_{\underline{\eta}}^{\hat{\eta}\left(\sigma^{\prime}\right)}\left[1-\beta\left(0 ; \eta, \sigma^{\prime}\right)\right] g(\eta) d \eta
$$

to

$$
\int_{\underline{\eta}}^{\hat{\eta}\left(\sigma^{\prime \prime}\right)}\left[1-\beta\left(0 ; \eta, \sigma^{\prime}\right)\right] g(\eta) d \eta .
$$

The distribution on discovered (and, for that matter, undiscovered) cartel duration shifts, in the short run, from

$$
\tilde{f}\left(l ; \sigma^{\prime}\right) \equiv \int_{\underline{\eta}}^{\hat{\eta}\left(\sigma^{\prime}\right)} f\left(l ; \eta, \sigma^{\prime}\right)\left[\frac{\left(1-\beta\left(0 ; \eta, \sigma^{\prime}\right)\right) g(\eta)}{\int_{\underline{\eta}}^{\hat{\eta}\left(\sigma^{\prime}\right)}\left[1-\beta\left(0 ; \eta^{\prime}, \sigma^{\prime}\right)\right] g\left(\eta^{\prime}\right) d \eta^{\prime}}\right] d \eta
$$

to

$$
\bar{f}\left(l ; \sigma^{\prime}, \sigma^{\prime \prime}\right) \equiv \int_{\underline{\eta}}^{\hat{\eta}\left(\sigma^{\prime \prime}\right)} f\left(l ; \eta, \sigma^{\prime}\right)\left[\frac{\left(1-\beta\left(0 ; \eta, \sigma^{\prime}\right)\right) g(\eta)}{\int_{\underline{\eta}}^{\hat{\eta}\left(\sigma^{\prime \prime}\right)}\left[1-\beta\left(0 ; \eta^{\prime}, \sigma^{\prime}\right)\right] g\left(\eta^{\prime}\right) d \eta^{\prime}}\right] d \eta .
$$

Notice that the relative weight on $f\left(l ; \eta, \sigma^{\prime}\right)$, for $\eta \in\left[\eta, \hat{\eta}\left(\sigma^{\prime \prime}\right)\right]$, is unchanged.

THEOREM 7. If $\sigma^{\prime \prime}>\sigma^{\prime}$ then $\bar{f}\left(l ; \sigma^{\prime}, \sigma^{\prime \prime}\right)$ first-order stochastically dominates (FOSD) $\tilde{f}\left(l ; \sigma^{\prime}\right)$. In other words, if a more aggressive detection and conviction policy has been effective then the duration of discovered cartels increases (in terms of FOSD) in the short run.

To summarize, a rise in $\sigma$ causes the immediate collapse of the least stable cartels (due to $\hat{\eta}$ being decreasing in $\sigma$ ). This means the surviving cartels are those with lower $\eta$ and thus longer duration. Because this is the pool from which one draws discovered cartels, the average duration of discovered cartels rises in the short run in response to a more aggressive detection and conviction policy. 
The transition from the short run to the (new) long run involves the distribution on cartel length shifting from

$$
\bar{f}\left(l ; \sigma^{\prime}, \sigma^{\prime \prime}\right)=\int_{\underline{\eta}}^{\hat{\eta}\left(\sigma^{\prime \prime}\right)} f\left(l ; \eta, \sigma^{\prime}\right)\left[\frac{\left(1-\beta\left(0 ; \eta, \sigma^{\prime}\right)\right) g(\eta)}{\int_{\underline{\eta}}^{\hat{\eta}\left(\sigma^{\prime \prime}\right)}\left[1-\beta\left(0 ; \eta^{\prime}, \sigma^{\prime}\right)\right] g\left(\eta^{\prime}\right) d \eta^{\prime}}\right] d \eta
$$

to

$$
\tilde{f}\left(l ; \sigma^{\prime \prime}\right)=\int_{\underline{\eta}}^{\hat{\eta}\left(\sigma^{\prime \prime}\right)} f\left(l ; \eta, \sigma^{\prime \prime}\right)\left[\frac{\left(1-\beta\left(0 ; \eta, \sigma^{\prime \prime}\right)\right) g(\eta)}{\int_{\underline{\eta}}^{\hat{\eta}\left(\sigma^{\prime \prime}\right)}\left[1-\beta\left(0 ; \eta^{\prime}, \sigma^{\prime \prime}\right)\right] g\left(\eta^{\prime}\right) d \eta^{\prime}}\right] d \eta .
$$

The latter is the stationary distribution on cartel duration when $\sigma=\sigma^{\prime \prime}$.

THEOREM 8. If $\sigma^{\prime \prime}>\sigma^{\prime}$ then $\bar{f}\left(l ; \sigma^{\prime}, \sigma^{\prime \prime}\right)$ FOSD $\tilde{f}\left(l ; \sigma^{\prime \prime}\right)$. In words, if a more aggressive detection and conviction policy has been effective then the duration of discovered cartels decreases (in terms of FOSD) as the industry goes from the short run to the long run.

In response to a policy that alters the likelihood of detection and conviction, its effect on the rate of cartels can be inferred by observing the duration of discovered cartels in the short run. If average cartel duration goes up (down) then the policy has caused $\sigma$ to rise (fall) and thus we can conclude that it will result in fewer (more) cartels forming in the new steady state.

\subsection{Robustness}

Let us explore robustness of the result that cartel duration rises in the short run in response to an increase in the probability of detection. The proof of that result relies on three steps. First, for a given competition policy, there is a cut-off $\hat{\eta}$ such that industries are capable of colluding if and only if $\eta \in[\eta, \hat{\eta}]$. Second, a more stringent competition policy (specifically, a higher value for $\sigma$ ) causes $\hat{\eta}$ to decline so that fewer industries are capable of sustaining a cartel. Third, the expected cartel duration is decreasing in $\eta$, which has the implication that the surviving cartels - after competition policy is made tougher-have longer duration. The cut-off property in the first step is due to the incremental value to colluding being decreasing in $\eta$. What is then essential is that when $\eta$ rises, both the incremental value to colluding and the expected cartel duration decline. Thus, in response to a tougher competition policy, it is the cartels with relatively high values for $\eta$ which collapse-as the incremental value to colluding was close enough to zero that it is now negative-and, in addition, those cartels have 
relatively short duration. In discussing other sources of heterogeneity, we will then explore the generality of the property that the incremental value to collusion and the expected cartel duration are positively correlated.

Suppose the source of industry heterogeneity is the discount factor, $\delta$, rather than the gains to price undercutting, $\eta$. An industry with more patient firms will obviously find it easier to satisfy the incentive compatibility constraints associated with collusion. This implies that cartel duration is longer for an industry with higher $\delta$, as it now takes a higher profit shock to destabilize the cartel. And because firms can expect to collude for a longer time once having cartelized, the incremental value to collusion is higher. Hence, there'll be some threshold industry type, denoted $\hat{\delta}$, such that a cartel is capable of forming if and only if $\delta>\hat{\delta}$. And when competition policy is made tougher, $\hat{\delta}$ will rise so that cartels with relatively low values of $\delta$ will collapse and those are also the cartels with relatively short duration. Again, we find that the surviving cartels tend to have longer duration, which implies the average duration of discovered cartels goes up.

Now suppose industry heterogeneity is in terms of the non-collusive solution, $\alpha$. As noted in footnote 10, the incremental value to collusion is decreasing in $\alpha$; if the non-collusive solution is less competitive, it is less valuable to collude. In Theorem 3, it is proven that $\varphi^{*}$ is decreasing in $\alpha$ and, because a lower value for $\varphi^{*}$ means a higher probability of cartel shutdown (as the cartel collapses whenever the realized profit shock is less than $\varphi^{*}$ ), expected cartel duration is decreasing in $\alpha$. Again, we find that those industries with lower incremental value to colluding have lower expected cartel duration.

It may be possible to construct other forms of heterogeneity whereby the incremental value to collusion and expected cartel duration are not positively correlated. For example, suppose industries differ in two dimensions: the profitability of the non-collusive outcome (which is controlled by $\alpha$ ) and the variability of the profit shock, which we will denote $\sigma_{\pi}^{2} \cdot{ }^{12}$ A higher value for $\sigma_{\pi}^{2}$ will tend to mean shorter cartel duration because it becomes more likely there will be a sufficiently high profit shock to cause the cartel to collapse. Now suppose $\alpha$ and $\sigma_{\pi}^{2}$ are negatively correlated so that industries which are not as competitive tend to have less variable profit shocks. It may then be the case that industries with lower incremental value to collusion (higher $\alpha$ ) have higher expected cartel duration (because $\sigma_{\pi}^{2}$ is lower). Hence, the more marginal cartels that shut down in response to a tougher competition policy may actually have higher cartel duration which means that the surviving cartels have lower duration and thus the average duration of discovered cartels will fall in response to a tougher competition policy. Though it does then appear there are forms of industry heterogeneity which could reverse the result, they are not likely to be common. And even in this carefully constructed case,

12. This example is due to the insightful comment of a referee. 
the result may not be reversed because longer average cartel duration, in and of itself, tends to raise the incremental value to collusion.

Rather than change the source of heterogeneity, another type of change is to allow an industry's profit state, $\pi$, to be persistent, rather than i.i.d., over time. Consider the extreme case in which profit is perfectly persistent but varies across industries. ${ }^{13}$ A cartel, once formed, will persist until it is discovered because there is no internal collapse since profit conditions do not change. It follows that the expected cartel duration is the same for all industry types which means that cartel duration is independent of competition policy (though the frequency of cartel formation is not). By a continuity argument, it would seem that the main result - cartel duration rises in the short run in response to an increase in $\sigma$-is weaker when profits are more persistent. Nevertheless, as long as profits are not too persistent, the result should hold though clearly the analysis would be more complex when there is the additional state variable in the form of the current profit state.

\subsection{Impact of a Corporate Leniency Program}

The previous analysis explored the impact of increasing penalties or making detection more likely. However, the most significant policy innovation in recent decades is the institution of a corporate leniency program, which brings forth a constellation of effects; some of which deter cartel formation (by making discovery more likely) and some of which encourage cartel formation (by waiving penalties). Here, we briefly review some results relevant to evaluating the efficacy of a leniency program. Using numerical analysis upon an expanded version of our model, we find - consistent with our earlier results - that a short-run rise in the average duration of discovered cartels indicates that a leniency program is working. 14

In modeling a leniency program, we have taken a standard approach in the literature (see Spagnolo 2008). If a single cartel member applies for leniency then the cartel is convicted for sure and the firm that applied receives a per period penalty of $\theta F$ where $\theta \in[0,1]$, whereas the other cartel members each pay $F$. As before, $F=\gamma(Y-\alpha \mu)$. Thus, a proportion $1-\theta$ of fines are waived for the firm that comes forward. If all firms simultaneously apply for leniency then each firm pays a penalty of $\omega F$, where $\omega \in(\theta, 1)$. For example, if only one firm can receive leniency and each firm has an equal probability of being first in the door then $\omega=(n-1+\theta) / n$ when there are $n$ cartel members. It is without

13. We thank a referee for making this insightful observation.

14. Details as to the model and the numerical analysis are provided in a supplemental appendix available from the authors. 
loss of generality that we only specify the leniency program when either one firm applies or all firms apply. Also, leniency is not awarded to firms that apply after another firm has done so, though the model could easily be adapted to allow for that modification.

There are various ways in which a leniency program affects the calculus to form and maintain a cartel. ${ }^{15}$ As described in Harrington (2008), the introduction of a leniency program can have three effects and these three effects are operative in our model. First, it can make cartels less stable (that is, the maximum profit state for cartel stability is lower) because a firm that cheats can, at the same time, apply for leniency and thus receive a higher payoff. Second, the probability of paying penalties is higher because firms in a collapsing cartel will find it optimal to apply for leniency. Specifically, the probability of discovery rises from $\sigma$ to $\sigma H\left(\varphi^{*}\right)+\left(1-H\left(\varphi^{*}\right)\right)$, where $1-H\left(\varphi^{*}\right)$ is the probability that a cartel collapses. Third, a leniency program affects the penalties that cartel members actually pay. When firms apply for leniency (which occurs in equilibrium when the cartel collapses), penalties are reduced from $F$ to $\omega F$. That effect actually serves to promote cartel formation. The introduction of a leniency program then has countervailing effects which makes a formal investigation worthwhile.

For all of the parameterizations of the model that we considered, a leniency program was found to be effective in reducing cartel formation.

PROPERTY 1. The introduction of a corporate leniency program reduces the long-run frequency of cartels and raises the rate at which cartels are discovered.

Of course, the population of cartels is not observed and thus those predictions cannot be tested directly. How the latent policy parameters $\theta$ and $\omega$ influence the observable population of discovered cartels is summarized as Property 2 . If in fact the leniency program is affecting cartel behavior as described in Property 1 then, in the short run, the duration of discovered cartels will rise. However, contrary to Theorem 8, the long-run effect on average observed cartel duration could go up or down.

PROPERTY 2. In the short run, the introduction of a corporate leniency program raises the average duration of discovered cartels. In the long run, the average duration of discovered cartels can go up or down.

\section{Concluding Remarks}

In the last 15 years, major policy changes in the manner in which cartels are discovered, prosecuted, and penalized have occurred in the European Union,

15. The ensuing discussion presumes leniency is sufficiently great (specifically, $\theta<\sigma$ ) so that it would be used by a cartel member. 
United States, and many other countries in the world. Though there are various intermediate measures of the impact of these policies-most notably the number of convictions, the number of leniency applications, and the penalties imposedthe true measure of interest is the number of cartels in existence. Success and failure of a policy is ultimately decided by the extent to which cartels are deterred from forming. To our knowledge, this paper is the first to develop a method for inferring the impact of policy on the latent cartel rate using the observed population of discovered cartels.

The efficacy of a policy that is intended to increase the severity of penalties can be measured by observing the change in the number of discovered cartels as it serves as a useful proxy for the number of cartels. That is not the case, however, with policies that affect the probability of discovery and prosecution or with the introduction of a leniency program. Our main finding is that if these policies have, in fact, been effective in reducing the rate of cartel formation then we ought to observe a short-run increase in the duration of discovered cartels. The duration of discovered cartels can then provide some information as to what is happening with the underlying rate of cartelization in the economy. Though the results are admittedly tentative in light of the simplicity of our model, it does show that theory can shed light on the impact of policy innovations and inject some needed substance into the policy debate.

There are many possible extensions of this framework. In markets lacking significant entry barriers, an important constraint on collusion is the prospect of either entry or expansion by small non-cartel members. Though the threat of entry was not a constraint for most of the markets controlled by the vitamins cartel, it was in the case of vitamin $\mathrm{C}$ where expansion by Chinese suppliers eventually disrupted collusion. One extension of our framework is to allow for industry heterogeneity with respect to entry barriers and then endogenize the effect of cartel formation on the number of firms. Another extension is to endogenize the probability of detection and prosecution by assuming that the authority is constrained in how many cartels it can discover and cases it can handle.

Currently, there is an active policy debate in many countries as to the design of anti-cartel policies. It is absolutely vital that economists play a role in that debate in order to ensure that sound policies are implemented and those policies that are implemented are properly evaluated as to their impact. This is an admittedly difficult exercise but the alternative is to allow the debate to be dominated by casual and potentially misleading measures such as the number of convictions. Indeed, one would hope that a highly successful policy would ultimately be measured by the absence of convictions because few cartels are forming. But then we're always left with distinguishing such an absence from simply an ineffective policy; that is, there are still plenty of cartels, we're just not catching and convicting them. It is the importance of making that distinction that motivates this line of work and 
it is our hope that this paper will encourage others to venture into this arena and make more progress on this important economic and policy issue.

\section{Appendix: Proofs}

\section{A.1. Proof of Theorem 1}

Recall that

$$
\begin{aligned}
\psi(Y)= & \int_{\underline{\pi}}^{\varphi(Y)}[(1-\delta) \pi+\delta Y-\delta \sigma(Y-W)] h(\pi) d \pi \\
& +\int_{\varphi(Y)}^{\bar{\pi}}[(1-\delta) \alpha \pi+\delta W] h(\pi) d \pi-\delta \sigma F
\end{aligned}
$$

where

$$
\begin{aligned}
\varphi(Y) & =\frac{\delta(1-\sigma)(1-\kappa)(Y-\alpha \mu)}{[1-\delta(1-\kappa)](\eta-1)}, \\
W & =\frac{(1-\kappa)(1-\delta) \alpha \mu+\kappa Y}{1-\delta(1-\kappa)}, \\
F & =\gamma(Y-\alpha \mu) .
\end{aligned}
$$

Because $Y \geq W$

$$
\begin{aligned}
\psi(\mu) \leq & \int_{\underline{\pi}}^{\varphi(\mu)}[(1-\delta) \pi+\delta \mu] h(\pi) d \pi \\
& +\int_{\varphi(\mu)}^{\bar{\pi}}[(1-\delta) \alpha \pi+\delta \mu] h(\pi) d \pi-\delta \sigma F \\
= & \mu-\int_{\varphi(\mu)}^{\bar{\pi}}(1-\delta)(1-\alpha) \pi h(\pi) d \pi-\delta \sigma F,
\end{aligned}
$$

and, therefore, $\psi(\mu)<\mu$. Given that $\psi(\alpha \mu)=\alpha \mu$, it follows that $\psi$ maps $[\alpha \mu, \mu]$ into itself as long as $\psi^{\prime}(Y) \geq 0$ for all $Y$ - which we now show to hold.

If $\varphi(Y)<\underline{\pi}$ then

$$
\psi(Y)=\int_{\underline{\pi}}^{\bar{\pi}}[(1-\delta) \alpha \pi+\delta W] h(\pi) d \pi-\delta \sigma F,
$$

and thus

$$
\psi^{\prime}(Y)=\int_{\underline{\pi}}^{\bar{\pi}} \delta \frac{\partial W}{\partial Y} h(\pi) d \pi=\frac{\delta \kappa}{1-\delta(1-\kappa)}>0
$$


If $\bar{\pi}<\varphi(Y)$ then

$$
\psi(Y)=\int_{\underline{\pi}}^{\bar{\pi}}[(1-\delta) \pi+\delta Y-\delta \sigma(Y-W)] h(\pi) d \pi-\delta \sigma \gamma(Y-\alpha \mu),
$$

and thus

$$
\psi^{\prime}(Y)=\delta\left[1-\sigma\left(1-\frac{\partial W}{\partial Y}\right)-\sigma \frac{\partial F}{\partial Y}\right]=\delta\left[(1-\sigma)\left(1-\frac{\partial W}{\partial Y}\right)+\frac{\partial W}{\partial Y}-\sigma \gamma\right],
$$

which is positive because $\partial W / \partial Y=\kappa /(1-\delta(1-\kappa)) \in(0,1)$ and it is assumed $\kappa /(1-\delta(1-\kappa))>\sigma \gamma$.

If we now suppose $\varphi(Y) \in(\underline{\pi}, \bar{\pi})$, then

$$
\begin{aligned}
\psi^{\prime}(Y)= & {[(1-\delta) \varphi(Y)+\delta Y-\delta \sigma(Y-W)] h(\varphi(Y)) \varphi^{\prime}(Y) } \\
& +\int_{\underline{\pi}}^{\varphi(Y)} \delta\left[1-\sigma\left(1-\frac{\partial W}{\partial Y}\right)\right] h(\pi) d \pi \\
& -[(1-\delta) \alpha \varphi(Y)+\delta W] h(\varphi(Y)) \varphi^{\prime}(Y)+\int_{\varphi(Y)}^{\bar{\pi}} \delta \frac{\partial W}{\partial Y} h(\pi) d \pi-\delta \sigma \frac{\partial F}{\partial Y} \\
= & {[(1-\delta)(1-\alpha) \varphi(Y)+\delta(1-\sigma)(Y-W)] h(\varphi(Y)) \varphi^{\prime}(Y) } \\
& +\int_{\underline{\pi}}^{\varphi(Y)} \delta(1-\sigma)\left(1-\frac{\partial W}{\partial Y}\right) h(\pi) d \pi+\delta\left(\frac{\partial W}{\partial Y}-\sigma \frac{\partial F}{\partial Y}\right),
\end{aligned}
$$

which is positive because $\partial W / \partial Y \in(0,1)$ and

$$
\frac{\partial W}{\partial Y}-\sigma \frac{\partial F}{\partial Y}=\frac{\kappa}{1-\delta(1-\kappa)}-\sigma \gamma>0 .
$$

\section{A.2. Proof of Theorem 2}

Let us first prove that a collusive solution exists for $\eta$ close to 1 . First note that if $Y-\alpha \mu>0$ then

$$
\lim _{\eta \rightarrow 1} \frac{\delta(1-\sigma)(1-\kappa)(Y-\alpha \mu)}{[1-\delta(1-\kappa)](\eta-1)}=+\infty .
$$

Hence, $\lim _{\eta \rightarrow 1} \varphi(Y, \eta)>\bar{\pi}$, which implies

$$
\begin{aligned}
\lim _{\eta \rightarrow 1} \psi(Y)= & \int_{\underline{\pi}}^{\bar{\pi}}\left[(1-\delta) \pi+\delta Y-\left(\frac{\delta \sigma(1-\kappa)(1-\delta)(Y-\alpha \mu)}{1-\delta(1-\kappa)}\right)\right] h(\pi) d \pi \\
& -\delta \sigma \gamma(Y-\alpha \mu) .
\end{aligned}
$$


Next note that

$$
\lim _{\sigma \rightarrow 0} \lim _{\eta \rightarrow 1} \psi(Y)=\int_{\underline{\pi}}^{\bar{\pi}}[(1-\delta) \pi+\delta Y] h(\pi) d \pi,
$$

where the order of limits does not matter. Recall that $(1-\delta) \mu+\delta Y>Y$ if and only if $Y<\mu$; therefore, if $Y \in(\alpha \mu, \mu)$, then $\lim _{\sigma \rightarrow 0} \lim _{\eta \rightarrow 1} \psi(Y)>Y$. Thus, if $Y<\mu$ then $\psi(Y)>Y$ for $\eta$ sufficiently close to one and $\sigma$ sufficiently close to zero. By continuity, there exists $Y \in(\alpha \mu, \mu]$ such that $\psi(Y)=Y$. We then have the following conclusion: If $\sigma$ is sufficiently close to zero then there exists $\hat{\eta}>1$ such that $Y^{*}(\eta) \in(\alpha \mu, \mu]$ for all $\eta \in(1, \hat{\eta}]$.

The next step is to prove that a collusive solution does not exist for large enough $\eta$. We want to show that $\psi(Y)<Y$ for all $Y>\alpha \mu$ when $\eta \rightarrow+\infty$. The ICC is

$$
(1-\delta) \pi+\delta(1-\sigma) Y+\delta \sigma(W-F) \geq(1-\delta) \eta \pi+\delta W-\delta \sigma F,
$$

which is equivalent to

$$
\delta(1-\sigma)(Y-W) \geq(1-\delta)(\eta-1) \pi,
$$

where the left-hand side is increasing in $Y$ and decreasing in $W$ and the right-hand side is increasing $\pi$. Because (a) $\pi \geq \underline{\pi}$ (>0), (b) $W \geq \alpha \mu$, and (c) $Y \leq \mu$, the following is a sufficient condition for equation (A.1) not to hold for all $\pi$ :

$$
\delta(1-\sigma)(1-\alpha) \mu<(1-\delta)(\eta-1) \underline{\pi},
$$

which is equivalent to

$$
\frac{(1-\delta) \underline{\pi}+\delta(1-\sigma)(1-\alpha) \mu}{(1-\delta) \underline{\pi}}<\eta .
$$

Thus, there exists $\eta^{\prime}$ such that a cartel never forms when $\eta>\eta^{\prime}$.

To sum, there exists $\hat{\eta}>1$ such that $Y^{*}(\eta) \in(\alpha \mu, \mu]$ for all $\eta \in(1, \hat{\eta}]$ and there exists $\eta^{\prime}>\hat{\eta}$ such that $\psi(Y ; \eta)<Y$ for all $Y>\alpha \mu$ and all $\eta>\eta^{\prime}$. Therefore, $Y^{*}(\eta)=\alpha \mu$ for all $\eta>\eta^{\prime}$. It is shown in the proof of Theorem 3 that $\psi(Y)$ is decreasing in $\eta$. Hence, if $\psi\left(Y ; \eta^{\prime \prime}\right)<Y$ for all $Y>\alpha \mu$ then $\psi(Y ; \eta)<Y$ for all $Y>\alpha \mu$ and all $\eta>\eta^{\prime \prime}$. We conclude that there is $\hat{\eta}>1$ such that $Y^{*}(\eta) \in(\alpha \mu, \mu]$ for all $\eta \in(1, \hat{\eta}]$ and $Y^{*}(\eta)=\alpha \mu$ for all $\eta>\hat{\eta}$.

\section{A.3. Proof of Theorem 3}

If $Y^{*}(\eta) \in\{\alpha \mu, \mu\}$ then the maximal fixed point is a corner solution, in which case, generically, marginal changes in parameters do not affect $Y^{*}(\eta)$. For the 
remainder of the proof suppose $Y^{*}(\eta) \in(\alpha \mu, \mu)$. Furthermore, assume initially that $\varphi \in(\underline{\pi}, \bar{\pi})$.

To explore the effect of $\sigma$ on $Y^{*}(\eta)$, consider

$$
\begin{aligned}
\frac{\partial \psi(Y)}{\partial \sigma}= & {[(1-\delta) \varphi+\delta Y-\delta \sigma(Y-W)] h(\varphi) \frac{\partial \varphi}{\partial \sigma} } \\
& -\int_{\underline{\pi}}^{\varphi} \delta(Y-W) h(\pi) d \pi-[(1-\delta) \alpha \varphi+\delta W] h(\varphi) \frac{\partial \varphi}{\partial \sigma}-\delta F \\
= & {[(1-\delta)(1-\alpha) \varphi+\delta(1-\sigma)(Y-W)] h(\varphi) \frac{\partial \varphi}{\partial \sigma} } \\
& -\int_{\underline{\pi}}^{\varphi} \delta(Y-W) h(\pi) d \pi-\delta F,
\end{aligned}
$$

which is negative because

$$
\frac{\partial \varphi}{\partial \sigma}=\frac{-\delta(1-\kappa)(Y-\alpha \mu)}{[1-\delta(1-\kappa)](\eta-1)}<0 .
$$

Now consider increasing $\sigma$ from $\sigma^{\prime}$ to $\sigma^{\prime \prime}$. Given that $\partial \psi(Y) / \partial \sigma<0, \psi(Y)$ shifts down. Because $\psi\left(Y, \sigma^{\prime}\right) \leq Y$ as $Y \geq Y^{*}\left(\sigma^{\prime}\right)$, we have $\psi\left(Y, \sigma^{\prime \prime}\right)<Y$ for all $Y \geq Y^{*}\left(\sigma^{\prime}\right)$; this implies $Y^{*}\left(\sigma^{\prime \prime}\right)<Y^{*}\left(\sigma^{\prime}\right)$. The other comparative statics on $Y^{*}$ will also use this method of showing how a change in a parameter affects $\psi(Y)$.

Next consider changing $\gamma$. Because

$$
\frac{\partial \psi(Y)}{\partial \gamma}=-\delta \sigma(Y-\alpha \mu)<0,
$$

$\psi(Y)$ is also decreasing in $\gamma$; this implies that $Y^{*}$ is decreasing in $\gamma$.

If instead $\varphi=\underline{\pi}$ then $Y^{*}(\eta)=\alpha \mu$ and thus $Y^{*}(\eta)$ is, generically, independent of $\sigma$ and $\gamma$. If $\varphi=\bar{\pi}$ then

$$
\begin{aligned}
\psi(Y)= & \int_{\underline{\pi}}^{\bar{\pi}}[(1-\delta) \pi+\delta Y-\delta \sigma(Y-W)] h(\pi) d \pi-\delta \sigma F \\
= & \int_{\underline{\pi}}^{\bar{\pi}}\left[(1-\delta) \pi+\delta Y-\frac{\delta \sigma(1-\kappa)(1-\delta)(Y-\alpha \mu)}{1-\delta(1-\kappa)}\right] h(\pi) d \pi \\
& -\delta \sigma \gamma(Y-\alpha \mu),
\end{aligned}
$$

which is decreasing in $\sigma$ and $\gamma$ and, therefore, $Y^{*}$ is decreasing in $\sigma$ and $\gamma$. 
Now consider the impact of changing $\eta$. Note that $\eta$ only operates through $\varphi$ since, in equilibrium, a firm never cheats. Hence, $Y^{*}(\eta)$ is independent of $\eta$ when $\varphi \notin(\underline{\pi}, \bar{\pi})$. Let us then suppose $\varphi \in(\underline{\pi}, \bar{\pi})$, in which case

$$
\begin{aligned}
& \frac{\partial \psi(Y)}{\partial \eta} \\
& \quad=[(1-\delta) \varphi+\delta Y-\delta \sigma(Y-W)] h(\varphi) \frac{\partial \varphi}{\partial \eta}-[(1-\delta) \alpha \varphi+\delta W] h(\varphi) \frac{\partial \varphi}{\partial \eta} \\
& \quad=[(1-\delta)(1-\alpha) \varphi+\delta(1-\sigma)(Y-W)] h(\varphi) \frac{\partial \varphi}{\partial \eta},
\end{aligned}
$$

which is negative because

$$
\frac{\partial \varphi}{\partial \eta}=-\frac{\delta(1-\sigma)(1-\kappa)(Y-\alpha \mu)}{[1-\delta(1-\kappa)](\eta-1)^{2}} .
$$

Hence, raising $\eta$ lowers $\psi(Y)$ and thus lowers $Y^{*}$.

Turning to comparative statics for $\varphi^{*}$, first note that if $\varphi^{*}>\underline{\pi}$ then $Y^{*}>\alpha \mu$ and

$$
\varphi^{*}=\frac{\delta(1-\sigma)(1-\kappa)\left(Y^{*}-\alpha \mu\right)}{[1-\delta(1-\kappa)](\eta-1)} .
$$

We will consider successively how $\varphi^{*}$ depends on $\sigma$, on $\gamma$, and on $\eta$-each time denoting as the argument of $\varphi^{*}$ and $Y^{*}$ the variable with respect to which comparative statics are being performed.

For $\sigma^{\prime \prime}>\sigma^{\prime}, \varphi^{*}\left(\sigma^{\prime \prime}\right)-\varphi^{*}\left(\sigma^{\prime}\right)$ is equal to

$$
\begin{aligned}
& \frac{\delta\left(1-\sigma^{\prime \prime}\right)(1-\kappa)\left(Y^{*}\left(\sigma^{\prime \prime}\right)-\alpha \mu\right)}{[1-\delta(1-\kappa)](\eta-1)}-\frac{\delta\left(1-\sigma^{\prime}\right)(1-\kappa)\left(Y^{*}\left(\sigma^{\prime}\right)-\alpha \mu\right)}{[1-\delta(1-\kappa)](\eta-1)} \\
= & \left(\frac{\delta(1-\kappa)}{[1-\delta(1-\kappa)](\eta-1)}\right) \\
& \quad \times\left[\left(1-\sigma^{\prime \prime}\right)\left(Y^{*}\left(\sigma^{\prime \prime}\right)-\alpha \mu\right)-\left(1-\sigma^{\prime}\right)\left(Y^{*}\left(\sigma^{\prime}\right)-\alpha \mu\right)\right],
\end{aligned}
$$

which is negative. Thus, $\varphi^{*}$ is decreasing in $\sigma$.

For $\gamma^{\prime \prime}>\gamma^{\prime}, \varphi^{*}\left(\gamma^{\prime \prime}\right)-\varphi^{*}\left(\gamma^{\prime}\right)$ is equal to

$$
\begin{aligned}
\frac{\delta(1-\sigma)(1-\kappa)\left(Y^{*}\left(\gamma^{\prime \prime}\right)-\alpha \mu\right)}{[1-\delta(1-\kappa)](\eta-1)}-\frac{\delta\left(1-\sigma^{\prime}\right)(1-\kappa)\left(Y^{*}\left(\gamma^{\prime}\right)-\alpha \mu\right)}{[1-\delta(1-\kappa)](\eta-1)} & \\
=\left(\frac{\delta(1-\sigma)(1-\kappa)}{[1-\delta(1-\kappa)](\eta-1)}\right)\left[Y^{*}\left(\gamma^{\prime \prime}\right)-Y^{*}\left(\gamma^{\prime}\right)\right]<0, &
\end{aligned}
$$

because, by Theorem 3, $Y^{*}$ is decreasing in $\gamma$. Thus, $\varphi^{*}$ is decreasing in $\gamma$. 
For $\eta^{\prime \prime}>\eta^{\prime}, \varphi^{*}\left(\eta^{\prime \prime}\right)-\varphi^{*}\left(\eta^{\prime}\right)$ is equal to

$$
\begin{aligned}
& \frac{\delta(1-\sigma)(1-\kappa)\left(Y^{*}\left(\eta^{\prime \prime}\right)-\alpha \mu\right)}{[1-\delta(1-\kappa)]\left(\eta^{\prime \prime}-1\right)}-\frac{\delta\left(1-\sigma^{\prime}\right)(1-\kappa)\left(Y^{*}\left(\eta^{\prime}\right)-\alpha \mu\right)}{[1-\delta(1-\kappa)]\left(\eta^{\prime}-1\right)} \\
& =\left(\frac{\delta(1-\sigma)(1-\kappa)}{[1-\delta(1-\kappa)]}\right)\left[\left(\frac{Y^{*}\left(\eta^{\prime \prime}\right)-\alpha \mu}{\eta^{\prime \prime}-1}\right)-\left(\frac{Y^{*}\left(\eta^{\prime}\right)-\alpha \mu}{\eta^{\prime}-1}\right)\right]<0,
\end{aligned}
$$

because (a) $Y^{*}$ is decreasing in $\eta$ (by Theorem 3), (b) $Y^{*}\left(\eta^{\prime}\right)-\alpha \mu>Y^{*}\left(\eta^{\prime \prime}\right)-$ $\alpha \mu>0$, and (c) $\eta^{\prime \prime}-1>\eta^{\prime}-1$. Thus, $\varphi^{*}$ is decreasing in $\eta$.

Finally, let us show that $\varphi^{*}$ is decreasing in $\alpha$ when $\varphi^{*} \in(\underline{\pi}, \bar{\pi})$. Recall that a fixed point is defined by

$$
\begin{aligned}
Y= & \int_{\underline{\pi}}^{\varphi(Y)}\left[(1-\delta) \pi+\delta Y-\left(\frac{\delta \sigma(1-\kappa)(1-\delta)(Y-\alpha \mu)}{1-\delta(1-\kappa)}\right)\right] h(\pi) d \pi \\
& +\int_{\varphi(Y)}^{\bar{\pi}}\left[(1-\delta) \alpha \pi+\delta\left(\frac{(1-\kappa)(1-\delta) \alpha \mu+\kappa Y}{1-\delta(1-\kappa)}\right)\right] h(\pi) d \pi \\
& -\delta \sigma \gamma(Y-\alpha \mu),
\end{aligned}
$$

where

$$
\varphi(Y)=\frac{\delta(1-\sigma)(1-\kappa)(Y-\alpha \mu)}{[1-\delta(1-\kappa)](\eta-1)} .
$$

Subtract $\alpha \mu$ from both sides and define $\Delta \equiv Y-\alpha \mu$, so that we now are looking for a fixed point in $\Delta$ of $\Delta=\Phi(\Delta, \alpha)$, where

$$
\begin{aligned}
\Phi(\Delta, \alpha) \equiv & \int_{\underline{\pi}}^{\varphi(\Delta)}\left[(1-\delta) \pi+\delta \Delta+\delta \alpha \mu-\left(\frac{\delta \sigma(1-\kappa)(1-\delta) \Delta}{1-\delta(1-\kappa)}\right)\right] h(\pi) d \pi \\
& +\int_{\varphi(\Delta)}^{\bar{\pi}}\left[(1-\delta) \alpha \pi+\delta\left(\frac{(1-\kappa)(1-\delta) \alpha \mu+\kappa \Delta}{1-\delta(1-\kappa)}\right)\right. \\
& \left.\quad+\frac{\delta \kappa \alpha \mu}{1-\delta(1-\kappa)}\right] h(\pi) d \pi-\delta \sigma \gamma \Delta-\alpha \mu \\
= & \int_{\underline{\pi}}^{\varphi(\Delta)}\left[(1-\delta) \pi+\delta \Delta-\left(\frac{\delta \sigma(1-\kappa)(1-\delta) \Delta}{1-\delta(1-\kappa)}\right)\right] h(\pi) d \pi \\
& +\int_{\underline{\pi}}^{\varphi(\Delta)} \delta \alpha \mu h(\pi) d \pi+\int_{\varphi(\Delta)}^{\bar{\pi}}\left(\frac{\delta \kappa \alpha \mu}{1-\delta(1-\kappa)}\right) h(\pi) d \pi \\
& +\int_{\varphi(\Delta)}^{\bar{\pi}}\left[(1-\delta) \alpha \pi+\delta\left(\frac{(1-\kappa)(1-\delta) \alpha \mu+\kappa \Delta}{1-\delta(1-\kappa)}\right)\right] h(\pi) d \pi \\
& -\delta \sigma \gamma \Delta-\alpha \mu .
\end{aligned}
$$


Note that $\varphi$, when written as a function of $\Delta$, does not depend directly on $\alpha$.

Let $\alpha=\alpha^{\prime}$ and suppose $\varphi^{*}\left(\eta ; \alpha^{\prime}\right) \in(\underline{\pi}, \bar{\pi})$ which implies that the maximal fixed point, $\Delta^{*}\left(\alpha^{\prime}\right)$, is interior and thus $\Phi\left(\Delta, \alpha^{\prime}\right) \leq 0$ as $\Delta \geq \Delta^{*}\left(\alpha^{\prime}\right)$. If we then show that $\Phi(\Delta, \alpha)$ is decreasing in $\alpha$, it follows that if $\alpha^{\prime \prime}>\alpha^{\prime}$ then $\Phi\left(\Delta, \alpha^{\prime \prime}\right)<0$ for all $\Delta \geq \Delta^{*}\left(\alpha^{\prime}\right)$, and therefore $\Delta^{*}\left(\alpha^{\prime \prime}\right)<\Delta^{*}\left(\alpha^{\prime}\right)$.

If $\varphi^{*}\left(\eta ; \alpha^{\prime}\right) \in(\underline{\pi}, \bar{\pi})$ then

$$
\begin{aligned}
\frac{\partial \Phi(\Delta, \alpha)}{\partial \alpha}= & \int_{\underline{\pi}}^{\varphi(\Delta)} \delta \mu h(\pi) d \pi+\int_{\varphi(\Delta)}^{\bar{\pi}}\left(\frac{\delta \kappa \mu}{1-\delta(1-\kappa)}\right) h(\pi) d \pi \\
& +\int_{\varphi(\Delta)}^{\bar{\pi}}\left[(1-\delta) \pi+\delta\left(\frac{(1-\kappa)(1-\delta) \mu}{1-\delta(1-\kappa)}\right)\right] h(\pi) d \pi-\mu \\
= & -\int_{\underline{\pi}}^{\varphi(\Delta)}(1-\delta) \pi h(\pi) d \pi<0 .
\end{aligned}
$$

We conclude that if $\varphi^{*}\left(\eta ; \alpha^{\prime}\right) \in(\underline{\pi}, \bar{\pi})$ then $\Delta^{*}\left(\alpha^{\prime}\right)$ is (locally) decreasing in $\alpha$.

Next note that

$$
\varphi^{*}(\eta ; \alpha)=\frac{\delta(1-\sigma)(1-\kappa)\left[Y^{*}(\eta ; \alpha)-\alpha \mu\right]}{[1-\delta(1-\kappa)](\eta-1)}=\frac{\delta(1-\sigma)(1-\kappa) \Delta^{*}(\alpha)}{[1-\delta(1-\kappa)](\eta-1)} .
$$

Hence, $\varphi^{*}(\eta ; \alpha)$ is decreasing in $\alpha$ because $\Delta^{*}(\alpha)$ is decreasing in $\alpha$.

\section{A.4. Proof of Theorem 4}

Recall that $\hat{\eta}$ satisfies the property,

$$
\begin{array}{ll}
Y^{*}(\eta)>\alpha \mu & \text { if } \eta \in[\underline{\eta}, \hat{\eta}], \\
Y^{*}(\eta)=\alpha \mu & \text { if } \eta>\hat{\eta} .
\end{array}
$$

By the continuity of $\psi(Y, \eta)$ with respect to $Y$ and $\eta$ and because $\psi(Y, \eta)$ is decreasing in $\eta$ (when $Y^{*}(\eta)>\alpha \mu$ ), it follows that $\psi(Y, \hat{\eta}) \leq Y$ for all $Y$ and $\psi\left(Y^{*}(\hat{\eta}), \hat{\eta}\right)=Y^{*}(\hat{\eta})$. With this property, let us now argue that $\hat{\eta}$ is decreasing in $\sigma$ and $\gamma$.

Consider raising $\sigma$ from $\sigma^{\prime}$ to $\sigma^{\prime \prime}$. Because $\psi(Y, \eta)$ is decreasing in $\sigma$ for $Y>\alpha \mu$ and because $\psi\left(Y, \hat{\eta}\left(\sigma^{\prime}\right), \sigma^{\prime}\right) \leq Y$ for all $Y$, we have $\psi\left(Y, \hat{\eta}\left(\sigma^{\prime}\right), \sigma^{\prime \prime}\right)<$ $Y$ for all $Y>\alpha \mu$. Given that $\psi(Y, \eta)$ is decreasing in $\eta$ for $Y>\alpha \mu$, we have $\hat{\eta}\left(\sigma^{\prime \prime}\right)<\hat{\eta}\left(\sigma^{\prime}\right)$. A similar argument applies to changes in $\gamma$.

To show that $\hat{\eta}$ is decreasing in $\alpha$, recall from the definition of $\hat{\eta}(\alpha)$ that $\Phi(\Delta, \hat{\eta}(\alpha), \alpha) \leq \Delta$ for all $\Delta$. Because $\Phi(\Delta, \hat{\eta}(\alpha), \alpha)$ is decreasing in $\alpha$ (holding $\hat{\eta}$ fixed), the following holds: if $\alpha^{\prime \prime}>\alpha^{\prime}$ then $\Phi\left(\Delta, \hat{\eta}\left(\alpha^{\prime}\right), \alpha^{\prime \prime}\right)<\Delta$ for all 
$\Delta>0$. This implies $\hat{\eta}\left(\alpha^{\prime \prime}\right)<\hat{\eta}\left(\alpha^{\prime}\right)$ if $\Phi\left(\Delta, \hat{\eta}\left(\alpha^{\prime}\right), \alpha^{\prime \prime}\right)$ is decreasing in $\eta$. Because $\Phi(\Delta, \eta, \alpha)=\psi(Y, \eta, \alpha)-\alpha \mu$ and we've already shown that $\psi(Y, \eta, \alpha)$ is decreasing in $\eta$, we have $\Phi(\Delta, \eta, \alpha)$ decreasing in $\eta$.

\section{A.5. Proof of Theorem 5}

Let us first consider the impact of $\gamma$ on the stationary rate of cartels for a type- $\eta$ sub-population. Suppose that penalties are made more severe as reflected in $\gamma$ being increased from $\gamma^{\prime}$ to $\gamma^{\prime \prime}$. As the cartel rate is

$$
\frac{\kappa(1-\sigma) H\left(\varphi^{*}(\gamma, \eta)\right)}{1-(1-\kappa)(1-\sigma) H\left(\varphi^{*}(\gamma, \eta)\right)},
$$

the change in the cartel rate is

$$
\frac{\kappa(1-\sigma) H\left(\varphi^{*}\left(\gamma^{\prime \prime}, \eta\right)\right)}{1-(1-\kappa)(1-\sigma) H\left(\varphi^{*}\left(\gamma^{\prime \prime}, \eta\right)\right)}-\frac{\kappa(1-\sigma) H\left(\varphi^{*}\left(\gamma^{\prime}, \eta\right)\right)}{1-(1-\kappa)(1-\sigma) H\left(\varphi^{*}\left(\gamma^{\prime}, \eta\right)\right)} .
$$

Next note that

$$
\begin{gathered}
\operatorname{sign}\left\{\frac{\kappa(1-\sigma) H\left(\varphi^{*}\left(\gamma^{\prime \prime}, \eta\right)\right)}{1-(1-\kappa)(1-\sigma) H\left(\varphi^{*}\left(\gamma^{\prime \prime}, \eta\right)\right)}-\frac{\kappa(1-\sigma) H\left(\varphi^{*}\left(\gamma^{\prime}, \eta\right)\right)}{1-(1-\kappa)(1-\sigma) H\left(\varphi^{*}\left(\gamma^{\prime}, \eta\right)\right)}\right\} \\
=\operatorname{sign}\left\{\kappa(1-\sigma)\left[H\left(\varphi^{*}\left(\gamma^{\prime \prime}, \eta\right)\right)-H\left(\varphi^{*}\left(\gamma^{\prime}, \eta\right)\right)\right]\right\}<0,
\end{gathered}
$$

because $\varphi^{*}\left(\gamma^{\prime \prime}\right)<\varphi^{*}\left(\gamma^{\prime}\right)$ by Theorem 3 .

It is then straightforward to extend this to the entire population of cartels. Recall that the cartel rate is

$$
\int_{\underline{\eta}}^{\hat{\eta}(\gamma)}\left[\frac{\kappa(1-\sigma) H\left(\varphi^{*}(\gamma, \eta)\right)}{1-(1-\kappa)(1-\sigma) H\left(\varphi^{*}(\gamma, \eta)\right)}\right] g(\eta) d \eta
$$

after integrating over all type- $\eta$ industries. It has just been shown that the integrand is decreasing in $\gamma$ and because $\hat{\eta}(\gamma)$ is decreasing in $\gamma$ by Theorem 4 then this expression is decreasing in $\gamma$. Because the rate of discovered cartels is proportional to the rate of cartels then the discovered cartel rate is also decreasing in $\gamma$.

\section{A.6. Proof of Theorem 6}

Suppose conviction is made more likely so that $\sigma$ is raised from $\sigma^{\prime}$ to $\sigma^{\prime \prime}$. Using equation (13), the change in the rate of cartels is

$$
\frac{\kappa\left(1-\sigma^{\prime \prime}\right) H\left(\varphi^{*}\left(\sigma^{\prime \prime}, \eta\right)\right)}{1-(1-\kappa)\left(1-\sigma^{\prime \prime}\right) H\left(\varphi^{*}\left(\sigma^{\prime \prime}, \eta\right)\right)}-\frac{\kappa\left(1-\sigma^{\prime}\right) H\left(\varphi^{*}\left(\sigma^{\prime}, \eta\right)\right)}{1-(1-\kappa)\left(1-\sigma^{\prime}\right) H\left(\varphi^{*}\left(\sigma^{\prime}, \eta\right)\right)} .
$$


The sign of that expression is the same as

$$
\begin{aligned}
\operatorname{sign}\left\{\kappa\left(1-\sigma^{\prime \prime}\right)\right. & H\left(\varphi^{*}\left(\sigma^{\prime \prime}, \eta\right)\right)\left[1-(1-\kappa)\left(1-\sigma^{\prime}\right) H\left(\varphi^{*}\left(\sigma^{\prime}, \eta\right)\right)\right] \\
\left.-\kappa\left(1-\sigma^{\prime}\right) H\left(\varphi^{*}\left(\sigma^{\prime}, \eta\right)\right)\left[1-(1-\kappa)\left(1-\sigma^{\prime \prime}\right) H\left(\varphi^{*}\left(\sigma^{\prime \prime}, \eta\right)\right)\right]\right\} & \operatorname{sign}\left\{\left(1-\sigma^{\prime \prime}\right) H\left(\varphi^{*}\left(\sigma^{\prime \prime}, \eta\right)\right)-\left(1-\sigma^{\prime}\right) H\left(\varphi^{*}\left(\sigma^{\prime}, \eta\right)\right)\right\}<0 .
\end{aligned}
$$

This expression is negative because $\sigma^{\prime \prime}>\sigma^{\prime}$ implies $1-\sigma^{\prime \prime}<1-\sigma^{\prime}$ and $H\left(\varphi^{*}\left(\sigma^{\prime \prime}, \eta\right)\right)<H\left(\varphi^{*}\left(\sigma^{\prime}, \eta\right)\right)$, since $\varphi^{*}\left(\sigma^{\prime \prime}, \eta\right)<\varphi^{*}\left(\sigma^{\prime}, \eta\right)$ by Theorem 3 .

It is then straightforward to extend this to the entire population of cartels. Recall that the cartel rate is

$$
\int_{\underline{\eta}}^{\hat{\eta}(\sigma)}\left[\frac{\kappa(1-\sigma) H\left(\varphi^{*}(\sigma, \eta)\right)}{1-(1-\kappa)(1-\sigma) H\left(\varphi^{*}(\sigma, \eta)\right)}\right] d G(\eta)
$$

after integrating over all type- $\eta$ industries. It has just been shown that the integrand is decreasing in $\sigma$ and since $\hat{\eta}(\sigma)$ is decreasing in $\sigma$ by Theorem 4 then this expression is decreasing in $\sigma$.

To show that the rate of discovered cartels can be either increasing or decreasing in $\sigma$, let us fix $\sigma^{\prime \prime}(>0)$ and make the initial probability of conviction sufficiently small ( $\sigma^{\prime} \simeq 0$ ). As $\sigma^{\prime} \rightarrow 0$, equation (15) holds because the righthand side goes to zero and the left-hand side is bounded above zero. Not surprisingly, the frequency of discovered cartels rises when the probability of conviction is raised from an initial level close to zero. Now suppose instead $\sigma^{\prime}$ is sufficiently small so that some cartels form; that is, $\hat{\eta}\left(\sigma^{\prime}\right)>\underline{\eta}$. Consider what happens when $\sigma^{\prime \prime}$ goes to one. Recall that $\psi(Y)$ is equal to

$$
\begin{aligned}
& \int_{\underline{\pi}}^{\frac{\delta(1-\sigma)(1-\kappa)(Y-\alpha \mu)}{[1-\delta(1-\kappa)](\eta-1)}}\left[(1-\delta) \pi+\delta Y-\left(\frac{\delta \sigma(1-\kappa)(1-\delta)(Y-\alpha \mu)}{1-\delta(1-\kappa)}\right)\right] h(\pi) d \pi \\
& \quad+\int_{\frac{\delta(1-\sigma)(1-\kappa)(Y-\alpha \mu)}{[1-\delta(1-\kappa)](\eta-1)}}^{\bar{\pi}}\left[(1-\delta) \alpha \pi+\delta\left(\frac{(1-\kappa)(1-\delta) \alpha \mu+\kappa Y}{1-\delta(1-\kappa)}\right)\right] h(\pi) d \pi \\
& \quad-\delta \sigma \gamma(Y-\alpha \mu) .
\end{aligned}
$$

For $Y-\alpha \mu>0$,

$$
\lim _{\sigma \rightarrow 1} \frac{\delta(1-\sigma)(1-\kappa)(Y-\alpha \mu)}{[1-\delta(1-\kappa)](\eta-1)}=0
$$


which implies

$$
\begin{aligned}
\lim _{\sigma \rightarrow 1} \psi(Y)= & \int_{\underline{\pi}}^{\bar{\pi}}\left[(1-\delta) \alpha \pi+\delta\left(\frac{(1-\kappa)(1-\delta) \alpha \mu+\kappa Y}{1-\delta(1-\kappa)}\right)\right] h(\pi) d \pi \\
& -\delta \gamma(Y-\alpha \mu) \\
= & (1-\delta) \alpha \mu+\delta\left(\frac{(1-\kappa)(1-\delta) \alpha \mu+\kappa Y}{1-\delta(1-\kappa)}\right)-\delta \gamma(Y-\alpha \mu) .
\end{aligned}
$$

If $\gamma=0$ then

$$
\begin{aligned}
\lim _{\sigma \rightarrow 1} \psi(Y) & =(1-\delta) \alpha \mu+\delta\left(\frac{(1-\kappa)(1-\delta) \alpha \mu+\kappa Y}{1-\delta(1-\kappa)}\right) \\
& =\frac{(1-\delta) \alpha \mu+\delta \kappa Y}{1-\delta(1-\kappa)} \in(\alpha \mu, Y) .
\end{aligned}
$$

Because $\psi(Y)$ is decreasing in $\gamma$, if $Y>\alpha \mu$ then $\lim _{\sigma \rightarrow 1} \psi(Y)<Y$. This implies $Y^{*}(\eta)=\alpha \mu$ for all $\eta>1$ as $\sigma \rightarrow 1$. Hence, $\lim _{\sigma \rightarrow 1} \hat{\eta}(\sigma)=1$ so the cartel rate is zero when $\sigma^{\prime \prime}$ is sufficiently close to one. Because $\beta\left(0 ; \eta, \sigma^{\prime \prime}\right)=1$ for all $\eta>1$, equation (15) does not hold since the right-hand side is positive and the left-hand side is zero.

\section{A.7. Proof of Theorem 7}

To prove Theorem 7, the next lemma will be useful. It shows for a type- $\eta$ subpopulation that a fall in $\sigma$ (and $\gamma$ ) causes a first-order stochastic dominance shift in the distribution over the length of cartels and discovered cartels.

Lemma 1. A decrease in $\sigma$ or $\gamma$ causes a first-order stochastic dominance (FOSD) shift in the stationary type- $\eta$ distribution on the duration of cartels and the duration of discovered cartels.

Proof. Suppose $\sigma$ is raised from $\sigma^{\prime}$ to $\sigma^{\prime \prime}$ and consider the impact of the proportion of cartels of length $l$.

$$
\begin{aligned}
\frac{f\left(l ; \eta, \sigma^{\prime \prime}\right)}{f\left(l ; \eta, \sigma^{\prime}\right)} & =\frac{H\left(\varphi^{*}\left(\sigma^{\prime \prime}\right)\right)^{l-1}\left(1-\sigma^{\prime \prime}\right)^{l-1}\left[1-H\left(\varphi^{*}\left(\sigma^{\prime \prime}\right)\right)\left(1-\sigma^{\prime \prime}\right)\right]}{H\left(\varphi^{*}\left(\sigma^{\prime}\right)\right)^{l-1}\left(1-\sigma^{\prime}\right)^{l-1}\left[1-H\left(\varphi^{*}\left(\sigma^{\prime}\right)\right)\left(1-\sigma^{\prime}\right)\right]} \\
& =\left(\frac{H\left(\varphi^{*}\left(\sigma^{\prime \prime}\right)\right)\left(1-\sigma^{\prime \prime}\right)}{H\left(\varphi^{*}\left(\sigma^{\prime}\right)\right)\left(1-\sigma^{\prime}\right)}\right)^{l-1}\left(\frac{1-H\left(\varphi^{*}\left(\sigma^{\prime \prime}\right)\right)\left(1-\sigma^{\prime \prime}\right)}{1-H\left(\varphi^{*}\left(\sigma^{\prime}\right)\right)\left(1-\sigma^{\prime}\right)}\right) .
\end{aligned}
$$

A more aggressive competition policy makes a duration of one period more likely:

$$
\frac{f\left(1 ; \eta, \sigma^{\prime \prime}\right)}{f\left(1 ; \eta, \sigma^{\prime}\right)}=\left(\frac{1-H\left(\varphi^{*}\left(\sigma^{\prime \prime}\right)\right)\left(1-\sigma^{\prime \prime}\right)}{1-H\left(\varphi^{*}\left(\sigma^{\prime}\right)\right)\left(1-\sigma^{\prime}\right)}\right)>1 \Rightarrow f\left(1 ; \eta, \sigma^{\prime \prime}\right)>f\left(1 ; \eta, \sigma^{\prime}\right) \text {. }
$$


Perform the following steps:

$$
\begin{aligned}
\frac{f\left(l ; \eta, \sigma^{\prime \prime}\right)}{f\left(l ; \eta, \sigma^{\prime}\right)}= & \left(\frac{H\left(\varphi^{*}\left(\sigma^{\prime \prime}\right)\right)\left(1-\sigma^{\prime \prime}\right)}{H\left(\varphi^{*}\left(\sigma^{\prime}\right)\right)\left(1-\sigma^{\prime}\right)}\right)^{l-1}\left(\frac{1-H\left(\varphi^{*}\left(\sigma^{\prime \prime}\right)\right)\left(1-\sigma^{\prime \prime}\right)}{1-H\left(\varphi^{*}\left(\sigma^{\prime}\right)\right)\left(1-\sigma^{\prime}\right)}\right) \\
\ln \left(\frac{f\left(l ; \eta, \sigma^{\prime \prime}\right)}{f\left(l ; \eta, \sigma^{\prime}\right)}\right)= & (l-1) \ln \left(\frac{H\left(\varphi^{*}\left(\sigma^{\prime \prime}\right)\right)\left(1-\sigma^{\prime \prime}\right)}{H\left(\varphi^{*}\left(\sigma^{\prime}\right)\right)\left(1-\sigma^{\prime}\right)}\right) \\
& +\ln \left(\frac{1-H\left(\varphi^{*}\left(\sigma^{\prime \prime}\right)\right)\left(1-\sigma^{\prime \prime}\right)}{1-H\left(\varphi^{*}\left(\sigma^{\prime}\right)\right)\left(1-\sigma^{\prime}\right)}\right) .
\end{aligned}
$$

Because

$$
\frac{\partial}{\partial l} \ln \left(\frac{f\left(l ; \eta, \sigma^{\prime \prime}\right)}{f\left(l ; \eta, \sigma^{\prime}\right)}\right)=\ln \left(\frac{H\left(\varphi^{*}\left(\sigma^{\prime \prime}\right)\right)\left(1-\sigma^{\prime \prime}\right)}{H\left(\varphi^{*}\left(\sigma^{\prime}\right)\right)\left(1-\sigma^{\prime}\right)}\right)<0
$$

and

$$
\frac{\partial}{\partial l} \ln \left(\frac{f\left(l ; \eta, \sigma^{\prime \prime}\right)}{f\left(l ; \eta, \sigma^{\prime}\right)}\right)=\left(\frac{\partial}{\partial l} \frac{f\left(l ; \eta, \sigma^{\prime \prime}\right)}{f\left(l ; \eta, \sigma^{\prime}\right)}\right) \frac{f\left(l ; \eta, \sigma^{\prime}\right)}{f\left(l ; \eta, \sigma^{\prime \prime}\right)}
$$

we have

$$
\frac{\partial}{\partial l} \frac{f\left(l ; \eta, \sigma^{\prime \prime}\right)}{f\left(l ; \eta, \sigma^{\prime}\right)}<0 .
$$

To summarize, $f\left(l ; \eta, \sigma^{\prime \prime}\right) / f\left(l ; \eta, \sigma^{\prime}\right)>1$ at $l=1$ and $f\left(l ; \eta, \sigma^{\prime \prime}\right) / f\left(l ; \eta, \sigma^{\prime}\right)$ is decreasing in $l$.

Because $\sum_{l=1}^{\infty} f(l ; \eta, \sigma)=1$, it cannot be the case that $f\left(l ; \eta, \sigma^{\prime \prime}\right) /$ $f\left(l ; \eta, \sigma^{\prime}\right)>1$ for some $l$ and $f\left(l ; \eta, \sigma^{\prime \prime}\right) / f\left(l ; \eta, \sigma^{\prime}\right) \geq 1$ for all $l$. It follows that, generically, there exists $l^{*} \geq 1$ such that $f\left(l ; \eta, \sigma^{\prime \prime}\right)>(<) f\left(l ; \eta, \sigma^{\prime}\right)$ as $l \geq(<) l^{*} \cdot f\left(l ; \eta, \sigma^{\prime}\right)$ then first-order stochastically dominates $f\left(l ; \eta, \sigma^{\prime \prime}\right)$.

A similar argument shows that lower penalties_-as reflected in reducing $\gamma$ from $\gamma^{\prime \prime}$ to $\gamma^{\prime}$-results in a FOSD shift of the distribution of cartel duration. First we have

$$
\begin{aligned}
\frac{f\left(l ; \eta, \gamma^{\prime \prime}\right)}{f\left(l ; \eta, \gamma^{\prime}\right)} & =\left(\frac{H\left(\varphi^{*}\left(\gamma^{\prime \prime}\right)\right)(1-\sigma)}{H\left(\varphi^{*}\left(\gamma^{\prime}\right)\right)(1-\sigma)}\right)^{l-1}\left(\frac{1-H\left(\varphi^{*}\left(\gamma^{\prime \prime}\right)\right)(1-\sigma)}{1-H\left(\varphi^{*}\left(\gamma^{\prime}\right)\right)(1-\sigma)}\right) \\
& =\left(\frac{H\left(\varphi^{*}\left(\gamma^{\prime \prime}\right)\right)}{H\left(\varphi^{*}\left(\gamma^{\prime}\right)\right)}\right)^{l-1}\left(\frac{1-H\left(\varphi^{*}\left(\gamma^{\prime \prime}\right)\right)(1-\sigma)}{1-H\left(\varphi^{*}\left(\gamma^{\prime}\right)\right)(1-\sigma)}\right) .
\end{aligned}
$$

For $l=1$, the first term equals 1 and so

$$
\frac{f\left(1 ; \eta, \gamma^{\prime \prime}\right)}{f\left(1 ; \eta, \gamma^{\prime}\right)}=\left(\frac{1-H\left(\varphi^{*}\left(\gamma^{\prime \prime}\right)\right)(1-\sigma)}{1-H\left(\varphi^{*}\left(\gamma^{\prime}\right)\right)(1-\sigma)}\right)>1,
$$


which implies $f\left(1 ; \eta, \gamma^{\prime \prime}\right)>f\left(1 ; \eta, \gamma^{\prime}\right)$. Furthermore

$$
\operatorname{sign}\left\{\frac{\partial}{\partial l} \frac{f\left(l ; \eta, \sigma^{\prime \prime}\right)}{f\left(l ; \eta, \sigma^{\prime}\right)}\right\}=\operatorname{sign}\left\{\frac{H\left(\varphi^{*}\left(\gamma^{\prime \prime}\right)\right)}{H\left(\varphi^{*}\left(\gamma^{\prime}\right)\right)}\right\}<0 .
$$

Hence, there exists $l^{*} \geq 1$ such that $f\left(l ; \eta, \gamma^{\prime \prime}\right)>(<) f\left(l ; \eta, \gamma^{\prime}\right)$ as $l \geq(<) l^{*}$, and, therefore, $f\left(l ; \eta, \gamma^{\prime}\right)$ FOSD $f\left(l ; \eta, \gamma^{\prime \prime}\right)$.

For the proof of Theorem 7 , we want to show that $\bar{f}\left(l ; \sigma^{\prime}, \sigma^{\prime \prime}\right)$ FOSD $\tilde{f}\left(l ; \sigma^{\prime}\right)$. That is, for any $l^{\prime}$,

$$
\begin{aligned}
& \sum_{l=1}^{l^{\prime}} \int_{\underline{\eta}}^{\hat{\eta}\left(\sigma^{\prime \prime}\right)} f\left(l ; \eta, \sigma^{\prime}\right)\left[\frac{\left(1-\beta\left(0 ; \eta, \sigma^{\prime}\right)\right) g(\eta)}{\int_{\underline{\eta}}^{\hat{\eta}\left(\sigma^{\prime \prime}\right)}\left[1-\beta\left(0 ; \eta^{\prime}, \sigma^{\prime}\right)\right] g\left(\eta^{\prime}\right) d \eta^{\prime}}\right] d \eta \\
& \quad<\sum_{l=1}^{l^{\prime}} \int_{\underline{\eta}}^{\hat{\eta}\left(\sigma^{\prime}\right)} f\left(l ; \eta, \sigma^{\prime}\right)\left[\frac{\left(1-\beta\left(0 ; \eta, \sigma^{\prime}\right)\right) g(\eta)}{\int_{\underline{\eta}}^{\hat{\eta}\left(\sigma^{\prime}\right)}\left[1-\beta\left(0 ; \eta^{\prime}, \sigma^{\prime}\right)\right] g\left(\eta^{\prime}\right) d \eta^{\prime}}\right] d \eta .
\end{aligned}
$$

Note that the term on the right of the inequality can be expressed as follows.

$$
\begin{aligned}
& \left(\frac{\int_{\underline{\eta}}^{\hat{\eta}\left(\sigma^{\prime \prime}\right)}\left[1-\beta\left(0 ; \eta^{\prime}, \sigma^{\prime}\right)\right] g\left(\eta^{\prime}\right) d \eta^{\prime}}{\int_{\underline{\eta}}^{\hat{\eta}\left(\sigma^{\prime}\right)}\left[1-\beta\left(0 ; \eta^{\prime}, \sigma^{\prime}\right)\right] g\left(\eta^{\prime}\right) d \eta^{\prime}}\right) \\
& \times \sum_{l=1}^{l^{\prime}} \int_{\underline{\eta}}^{\hat{\eta}\left(\sigma^{\prime \prime}\right)} f\left(l ; \eta, \sigma^{\prime}\right)\left[\frac{\left(1-\beta\left(0 ; \eta, \sigma^{\prime}\right)\right) g(\eta)}{\int_{\underline{\eta}}^{\hat{\eta}\left(\sigma^{\prime \prime}\right)}\left[1-\beta\left(0 ; \eta^{\prime}, \sigma^{\prime}\right)\right] g\left(\eta^{\prime}\right) d \eta^{\prime}}\right] d \eta \\
& +\left[\frac{\int_{\underline{\eta}}^{\hat{\eta}\left(\sigma^{\prime}\right)}\left[1-\beta\left(0 ; \eta^{\prime}, \sigma^{\prime}\right)\right] g\left(\eta^{\prime}\right) d \eta^{\prime}-\int_{\underline{\eta}}^{\hat{\eta}\left(\sigma^{\prime \prime}\right)}\left[1-\beta\left(0 ; \eta^{\prime}, \sigma^{\prime}\right)\right] g\left(\eta^{\prime}\right) d \eta^{\prime}}{\int_{\underline{\eta}}^{\hat{\eta}\left(\sigma^{\prime}\right)}\left[1-\beta\left(0 ; \eta^{\prime}, \sigma^{\prime}\right)\right] g\left(\eta^{\prime}\right) d \eta^{\prime}}\right) \\
& \times \sum_{l=1}^{l^{\prime}} \int_{\hat{\eta}\left(\sigma^{\prime \prime}\right)}^{\hat{\eta}\left(\sigma^{\prime}\right)} f\left(l ; \eta, \sigma^{\prime}\right) \\
& \times\left[\frac{\left(1-\beta\left(0 ; \eta, \sigma^{\prime}\right)\right) g(\eta)}{\int_{\underline{\eta}}^{\hat{\eta}\left(\sigma^{\prime}\right)}\left[1-\beta\left(0 ; \eta^{\prime}, \sigma^{\prime}\right)\right] g\left(\eta^{\prime}\right) d \eta^{\prime}-\int_{\underline{\eta}}^{\hat{\eta}\left(\sigma^{\prime \prime}\right)}\left[1-\beta\left(0 ; \eta^{\prime}, \sigma^{\prime}\right)\right] g\left(\eta^{\prime}\right) d \eta^{\prime}}\right] d \eta .
\end{aligned}
$$

What we've done is to break apart the integration into $\int_{\underline{\eta}}^{\hat{\eta}\left(\sigma^{\prime \prime}\right)}$ and $\int_{\hat{\eta}\left(\sigma^{\prime \prime}\right)}^{\hat{\eta}\left(\sigma^{\prime}\right)}$ and then multiplied the first term by

$$
\frac{\int_{\underline{\eta}}^{\hat{\eta}\left(\sigma^{\prime \prime}\right)}\left[1-\beta\left(0 ; \eta^{\prime}, \sigma^{\prime}\right)\right] g\left(\eta^{\prime}\right) d \eta^{\prime}}{\int_{\underline{\eta}}^{\hat{\eta}\left(\sigma^{\prime \prime}\right)}\left[1-\beta\left(0 ; \eta^{\prime}, \sigma^{\prime}\right)\right] g\left(\eta^{\prime}\right) d \eta^{\prime}}
$$


and the second term by

$$
\frac{\int_{\underline{\eta}}^{\hat{\eta}\left(\sigma^{\prime}\right)}\left[1-\beta\left(0 ; \eta^{\prime}, \sigma^{\prime}\right)\right] g\left(\eta^{\prime}\right) d \eta^{\prime}-\int_{\underline{\eta}}^{\hat{\eta}\left(\sigma^{\prime \prime}\right)}\left[1-\beta\left(0 ; \eta^{\prime}, \sigma^{\prime}\right)\right] g\left(\eta^{\prime}\right) d \eta^{\prime}}{\int_{\underline{\eta}}^{\hat{\eta}\left(\sigma^{\prime}\right)}\left[1-\beta\left(0 ; \eta^{\prime}, \sigma^{\prime}\right)\right] g\left(\eta^{\prime}\right) d \eta^{\prime}-\int_{\underline{\eta}}^{\hat{\eta}\left(\sigma^{\prime \prime}\right)}\left[1-\beta\left(0 ; \eta^{\prime}, \sigma^{\prime}\right)\right] g\left(\eta^{\prime}\right) d \eta^{\prime}} .
$$

Substituting equation (A.3) into equation (A.2), we then need to show

$$
\begin{aligned}
& \sum_{l=1}^{l^{\prime}} \int_{\underline{\eta}}^{\hat{\eta}\left(\sigma^{\prime \prime}\right)} f\left(l ; \eta, \sigma^{\prime}\right)\left[\frac{\left(1-\beta\left(0 ; \eta, \sigma^{\prime}\right)\right) g(\eta)}{\int_{\underline{\eta}}^{\hat{\eta}\left(\sigma^{\prime \prime}\right)}\left[1-\beta\left(0 ; \eta^{\prime}\right)\right] g\left(\eta^{\prime}\right) d \eta^{\prime}}\right] d \eta \\
& <v \sum_{l=1}^{l^{\prime}} \int_{\underline{\eta}}^{\hat{\eta}\left(\sigma^{\prime \prime}\right)} f\left(l ; \eta, \sigma^{\prime}\right)\left[\frac{\left(1-\beta\left(0 ; \eta, \sigma^{\prime}\right)\right) g(\eta)}{\int_{\underline{\eta}}^{\hat{\eta}\left(\sigma^{\prime \prime}\right)}\left[1-\beta\left(0 ; \eta^{\prime}, \sigma^{\prime}\right)\right] g\left(\eta^{\prime}\right) d \eta^{\prime}}\right] d \eta \\
& +(1-v) \sum_{l=1}^{l^{\prime}} \int_{\hat{\eta}\left(\sigma^{\prime \prime}\right)}^{\hat{\eta}\left(\sigma^{\prime}\right)} f\left(l ; \eta, \sigma^{\prime}\right) \times \\
& {\left[\frac{\left(1-\beta\left(0 ; \eta, \sigma^{\prime}\right)\right) g(\eta)}{\int_{\underline{\eta}}^{\hat{\eta}\left(\sigma^{\prime}\right)}\left[1-\beta\left(0 ; \eta^{\prime}, \sigma^{\prime}\right)\right] g\left(\eta^{\prime}\right) d \eta^{\prime}-\int_{\underline{\eta}}^{\hat{\eta}\left(\sigma^{\prime \prime}\right)}\left[1-\beta\left(0 ; \eta^{\prime}, \sigma^{\prime}\right)\right] g\left(\eta^{\prime}\right) d \eta^{\prime}}\right] d \eta,}
\end{aligned}
$$

where

$$
v \equiv \frac{\int_{\underline{\eta}}^{\hat{\eta}\left(\sigma^{\prime \prime}\right)}\left[1-\beta\left(0 ; \eta^{\prime}, \sigma^{\prime}\right)\right] g\left(\eta^{\prime}\right) d \eta^{\prime}}{\int_{\underline{\eta}}^{\hat{\eta}\left(\sigma^{\prime}\right)}\left[1-\beta\left(0 ; \eta^{\prime}, \sigma^{\prime}\right)\right] g\left(\eta^{\prime}\right) d \eta^{\prime}}
$$

Manipulating equation (A.4),

$$
\begin{aligned}
& (1-v) \sum_{l=1}^{l^{\prime}} \int_{\underline{\eta}}^{\hat{\eta}\left(\sigma^{\prime \prime}\right)} f\left(l ; \eta, \sigma^{\prime}\right)\left[\frac{\left(1-\beta\left(0 ; \eta, \sigma^{\prime}\right)\right) g(\eta)}{\int_{\underline{\eta}}^{\hat{\eta}\left(\sigma^{\prime \prime}\right)}\left[1-\beta\left(0 ; \eta^{\prime}\right)\right] g\left(\eta^{\prime}\right) d \eta^{\prime}}\right] d \eta \\
& <(1-v) \sum_{l=1}^{l^{\prime}} \int_{\hat{\eta}\left(\sigma^{\prime \prime}\right)}^{\hat{\eta}\left(\sigma^{\prime}\right)} f\left(l ; \eta, \sigma^{\prime}\right) \\
& \times\left[\frac{\left(1-\beta\left(0 ; \eta, \sigma^{\prime}\right)\right) g(\eta)}{\int_{\underline{\eta}}^{\hat{\eta}\left(\sigma^{\prime}\right)}\left[1-\beta\left(0 ; \eta^{\prime}, \sigma^{\prime}\right)\right] g\left(\eta^{\prime}\right) d \eta^{\prime}-\int_{\underline{\eta}}^{\hat{\eta}\left(\sigma^{\prime \prime}\right)}\left[1-\beta\left(0 ; \eta^{\prime}, \sigma^{\prime}\right)\right] g\left(\eta^{\prime}\right) d \eta^{\prime}}\right] d \eta,
\end{aligned}
$$


which is equvalent to

$$
\begin{aligned}
& \sum_{l=1}^{l^{\prime}} \int_{\underline{\eta}}^{\hat{\eta}\left(\sigma^{\prime \prime}\right)} f\left(l ; \eta, \sigma^{\prime}\right)\left[\frac{\left(1-\beta\left(0 ; \eta, \sigma^{\prime}\right)\right) g(\eta)}{\int_{\underline{\eta}}^{\hat{\eta}\left(\sigma^{\prime \prime}\right)}\left[1-\beta\left(0 ; \eta^{\prime}\right)\right] g\left(\eta^{\prime}\right) d \eta^{\prime}}\right] d \eta \\
& <\sum_{l=1}^{l^{\prime}} \int_{\hat{\eta}\left(\sigma^{\prime \prime}\right)}^{\hat{\eta}\left(\sigma^{\prime}\right)} f\left(l ; \eta, \sigma^{\prime}\right) \\
& \times\left[\frac{\left(1-\beta\left(0 ; \eta, \sigma^{\prime}\right)\right) g(\eta)}{\int_{\underline{\eta}}^{\hat{\eta}\left(\sigma^{\prime}\right)}\left[1-\beta\left(0 ; \eta^{\prime}, \sigma^{\prime}\right)\right] g\left(\eta^{\prime}\right) d \eta^{\prime}-\int_{\underline{\eta}}^{\hat{\eta}\left(\sigma^{\prime \prime}\right)}\left[1-\beta\left(0 ; \eta^{\prime}, \sigma^{\prime}\right)\right] g\left(\eta^{\prime}\right) d \eta^{\prime}}\right] d \eta
\end{aligned}
$$

Recall that if $\eta^{\prime \prime}>\eta^{\prime}$ then $f\left(l ; \eta^{\prime}, \sigma\right)$ FOSD $f\left(l ; \eta^{\prime \prime}, \sigma\right)$ (Lemma 1). Hence, except for $\eta=\hat{\eta}\left(\sigma^{\prime \prime}\right), f\left(l ; \eta^{\prime}, \sigma^{\prime}\right)<f\left(l ; \eta^{\prime \prime}, \sigma^{\prime}\right)$ for all $\eta^{\prime} \in \eta \in\left[\eta, \hat{\eta}\left(\sigma^{\prime \prime}\right)\right]$ and all $\eta^{\prime \prime} \in \eta\left[\hat{\eta}\left(\sigma^{\prime \prime}\right), \hat{\eta}\left(\sigma^{\prime}\right)\right]$. It follows that (A.5) holds.

\section{A.8. Proof of Theorem 8}

Because $f\left(l ; \eta, \sigma^{\prime}\right)$ FOSD $f\left(l ; \eta, \sigma^{\prime \prime}\right)$ for all $\eta \in\left[\eta, \hat{\eta}\left(\sigma^{\prime \prime}\right)\right]$ (by Lemma 1$)$, the short-run distribution on cartel duration FOSD the long-run distribution.

\section{References}

Chen, Joe, and Joseph E. Harrington, Jr. (2007). "The Impact of the Corporate Leniency Program on Cartel Formation and the Cartel Price Path." In The Political Economy of Antitrust, edited by Vivek Ghosal and Johan Stennek. Elsevier.

Connor, John M. (2007). "A Critique of Partial Leniency for Cartels by the U.S. Department of Justice.” Working paper, Purdue University.

Cyrenne, Philippe (1999). "On Antitrust Enforcement and the Deterrence of Collusive Behavior." Review of Industrial Organization, 14, 257-272.

Harrington, Joseph E., Jr. (2004). "Cartel Pricing Dynamics in the Presence of an Antitrust Authority." RAND Journal of Economics, 35, 651-673.

Harrington, Joseph E., Jr. (2005). "Optimal Cartel Pricing in the Presence of an Antitrust Authority." International Economic Review, 46, 145-169.

Harrington, Joseph E., Jr. (2008). "Optimal Corporate Leniency Programs." Journal of Industrial Economics, 56, 215-246.

Harrington, Joseph E., Jr., and Joe Chen (2006). "Cartel Pricing Dynamics with Cost Variability and Endogenous Buyer Detection.” International Journal of Industrial Organization, 24, $1185-1212$.

Levenstein, Margaret C., and Valerie Y. Suslow (2006). "Determinants of International Cartel Duration and the Role of Cartel Organization." Ross School of Business Paper No. 1052, University of Michigan.

Miller, Nathan H. (2009). "Strategic Leniency and Cartel Enforcement." American Economic Review, 99, 750-768. 
Rotemberg, Julio J., and Garth Saloner (1986). "A Supergame-Theoretic Model of Price Wars During Booms." American Economic Review, 76, 390-407.

Selten, Reinhard (1973). "A Simple Model of Imperfect Competition, Where 4 are Few and 6 are Many." International Journal of Game Theory, 2, 141-201.

Spagnolo, Giancarlo (2000). "Self-Defeating Antitrust Laws: How Leniency Programs Solve Bertrand's Paradox and Enforce Collusion in Auctions." FEEM Working Paper No. 52.2000.

Spagnolo, Giancarlo (2008). "Leniency and Whistleblowers in Antitrust." In Handbook of Antitrust Economics, edited by Paolo Buccirossi. The MIT Press.

Wils, Wouter P. J. (2007). “The European Commission's 2006 Guidelines on Antitrust Fines: A Legal and Economic Analysis." World Competition Law and Economics Review, 30, 197-229. 